\title{
Comparative fecal metagenomics unveils unique functional capacity of the swine gut
}

\author{
Regina Lamendella ${ }^{1,4}$, Jorge W Santo Domingo ${ }^{2^{*}}$, Shreya Ghosh', John Martinson ${ }^{3}$ and Daniel B Oerther ${ }^{1,5}$
}

\begin{abstract}
Background: Uncovering the taxonomic composition and functional capacity within the swine gut microbial consortia is of great importance to animal physiology and health as well as to food and water safety due to the presence of human pathogens in pig feces. Nonetheless, limited information on the functional diversity of the swine gut microbiome is available.

Results: Analysis of 637, 722 pyrosequencing reads (130 megabases) generated from Yorkshire pig fecal DNA extracts was performed to help better understand the microbial diversity and largely unknown functional capacity of the swine gut microbiome. Swine fecal metagenomic sequences were annotated using both MG-RAST and JGl IMG/M-ER pipelines. Taxonomic analysis of metagenomic reads indicated that swine fecal microbiomes were dominated by Firmicutes and Bacteroidetes phyla. At a finer phylogenetic resolution, Prevotella spp. dominated the swine fecal metagenome, while some genes associated with Treponema and Anareovibrio species were found to be exclusively within the pig fecal metagenomic sequences analyzed. Functional analysis revealed that carbohydrate metabolism was the most abundant SEED subsystem, representing 13\% of the swine metagenome. Genes associated with stress, virulence, cell wall and cell capsule were also abundant. Virulence factors associated with antibiotic resistance genes with highest sequence homology to genes in Bacteroidetes, Clostridia, and Methanosarcina were numerous within the gene families unique to the swine fecal metagenomes. Other abundant proteins unique to the distal swine gut shared high sequence homology to putative carbohydrate membrane transporters.

Conclusions: The results from this metagenomic survey demonstrated the presence of genes associated with resistance to antibiotics and carbohydrate metabolism suggesting that the swine gut microbiome may be shaped by husbandry practices.
\end{abstract}

\section{Background}

The animal gastrointestinal tract harbors a complex microbial network and its composition reflects the constant co-evolution of these microorganisms with their host environment [1]. Uncovering the taxonomic composition and functional capacity within the animal gut microbial consortia is of great importance to understanding the roles they play in the host physiology and health. Since animal feces can harbor human pathogens, understanding the genetic composition of fecal microbial communities also has important implications for food and water safety. The structure and function of the

\footnotetext{
* Correspondence: santodomingo.jorge@epa.gov

${ }^{2}$ National Risk Management Research Laboratory, U. S. Environmental

Protection Agency, Cincinnati, OH 45268, USA

Full list of author information is available at the end of the article
}

gut microbial community has received significant attention for decades, although most of the work was restricted by the use of culture-based techniques. Recently, sequence analysis of the 16S rRNA gene has shed new light on the diversity and composition of microbial communities within several animal gut systems [2]. While 16S rRNA gene-based techniques have revealed impressive microbial diversity within gut environments, this approach offers only limited information on the physiological role of microbial consortia within a given gut environment.

Random sequencing of metagenomes has allowed scientists to reveal significant differences in metabolic potential within different environments [3], including microbial populations associated with host-microbial partnerships. Specifically, the publicly available database
C Biomed Central

(c) 2011 Lamendella et al; licensee BioMed Central Ltd. This is an Open Access article distributed under the terms of the Creative Commons Attribution License (http://creativecommons.org/licenses/by/2.0), which permits unrestricted use, distribution, and reproduction in any medium, provided the original work is properly cited. 
IMG/M [4] contains $596 \mathrm{Mb}$ of sequencing data, representing 1,424, 000 genes from 17 different gut microbiomes. Studying gut metagenomes has particularly helped in uncovering several important biological characteristics of these microbiomes. For example, when 13 human gut metagenomes were compared, Kurokawa et al [5] found that adult and infant type gut microbiomes have enriched gene families sharing little overlap, suggesting different core functions within the adult and infantile gut microbiota. This study also demonstrated the presence of hundreds of gene families exclusively found in the adult human gut, suggesting various strategies are employed by each type of microbiota to adapt to its intestinal environment [5]. Other gut microbiome studies support these significant differences in core and variable gene content from different animal hosts and environments [1,6-12]. Thus, comparing the gene content of multiple gut microbiomes can help elucidate the ecological underpinnings of gut systems.

Thus far, the functional genetic potential of the pig distal gut microbiota has not been studied using metagenomics, although it is reasonable to assume that the swine gut supports similar genetic complexity to the human gut system, as they both prefer omnivorous feeding behavior and harbor similar bacterial groups as determined by several phylogenetic studies [13-15]. In this study we used metagenomic data analyses to characterize the swine fecal microbiome with respect to species composition and functional content. In order to search for the potential presence of unique gene functions harbored by the swine gut microbiome, we performed a comparative metagenomic approach, in the context of phylogenetic and functional composition.

\section{Results}

\section{Taxonomic distribution of swine fecal metagenomic} sequences

Approximately $130 \mathrm{Mb}$ of swine fecal metagenome sequence data were retrieved using two different pyrosequencing platforms (454 GS20 and FLX), making this study the first metagenomic survey of the swine gut (Table 1). The average read length for the GS20 and FLX runs were $156 \mathrm{bp}$ and $230 \mathrm{bp}$, respectively. Taxonomic distribution of 16S rRNA gene sequences from the GS20 and FLX swine fecal metagenomes revealed similar taxonomic distributions (Figure 1). However, some differences in classification of $16 \mathrm{~S}$ rRNA genes retrieved from the GS20 and FLX runs were noted. Most interestingly, fewer Firmicutes and more Bacteroidetes were classified using the FLX 16S rRNA genes (using both RDP and Greengenes databases). This finding suggests shorter read lengths may lead to misclassification of these two divergent phyla. Additionally, more unclassified sequences were retrieved from GS20 metagenomic reads with e-values less than 0.01 .

Both GS20 and FLX metagenomic swine fecal datasets were dominated by Firmicutes and Bacteroidetes phyla (Figure 1), which is consistent with several molecular phylogenetic studies of mammalian gut environments, including the swine gut $[2,8,10,14]$. Archaeal sequences constituted less than $1 \%$ of total rRNA gene sequences retrieved in either swine metagenome, and were dominated by the Methanomicrobia and Thermococci, which is consistent with previous molecular diversity studies of pig manure [16]. While these populations are only a very small fraction of the total microbiota [17], methanogens contribute significantly to the metabolic potential within in a gut environment [18]. The majority of eukaryotic sequences derived from the swine metagenomes are related to Chordata (i.e., host phylum), fungi, and the Viridiplantae (i.e., feed). Sequences sharing high sequence homology to Balantidium coli were obtained in both swine metagenomes. The latter is a protozoan pathogen that causes balantadiasis in mammalian hosts, including human and swine. Since the samples were collected from healthy animals, these sequences might be associated with non-pathogenic $B$. coli strains or with pathogenic strains in asymptomatic animals. Viral sequences were rare, comprising less than $1 \%$ of the total metagenomic sequences when compared to the SEED database (Additional File 1, Fig. S1). The low abundance of viral sequences retrieved from the swine fecal metagenomes is consistent with viral proportions retrieved in termite, chicken, and cattle gastrointestinal metagenomes, and may be a direct result of limited representation of viral genetic information in currently available databases [8].

A closer look at the taxonomic distribution of the numerically abundant bacterial orders derived from the swine metagenomes revealed that Clostridiales, unclassified Firmicutes, Bacteroidales, Spirochaetales, unclassified gammaproteobacteria, and Lactobacillales were the top six most abundant bacterial groups (Additional File 1, Fig. S2). At the genus-level taxonomic resolution, Prevotella species were the most abundant, comprising 19$22 \%$ of $16 \mathrm{~S}$ rRNA gene sequences within both swine fecal metagenomes (Additional File 1, Fig. S3). Of the classified Clostridiales, Sporobacter was the next most abundant genus within both the swine fecal metagenomic datasets. Anaerovibro, Clostridium, and Streptococcus genera encompassed at least 5\% of rRNA gene sequences in either the GS20 or FLX datasets.

\section{Comparative gut metagenomics using 16S rRNA gene sequences}

We performed comparative metagenomics on $16 \mathrm{~S}$ rRNA gene sequences derived from publicly available gut 
Table 1 Summary of pyrosequencing data from Yorkshire swine fecal samples

\begin{tabular}{|c|c|c|}
\hline & Yorkshire Pig Fecal Metagenome GS20 & Yorkshire Pig Fecal Metagenome FLX \\
\hline Total no. of sequences & 157,221 & 462,501 \\
\hline Total sequence size (bp) & $24,518,676$ & $106,193,719$ \\
\hline Average sequence length (bp) & 155.95 & 229.61 \\
\hline Genes* & 42677 & 124684 \\
\hline CDS* & $42349(99.23 \%)$ & $124050(99.49 \%)$ \\
\hline RNA* & $328(.77 \%)$ & $634(.51 \%)$ \\
\hline rRNA* & 328 & 634 \\
\hline $5 S$ & 25 & 46 \\
\hline $16 S$ & 114 & 248 \\
\hline $18 \mathrm{~S}$ & 1 & 2 \\
\hline $23 S$ & 181 & 325 \\
\hline $28 \mathrm{~S}$ & 1 & 3 \\
\hline Ribosomal Database Project $16 \mathrm{~S}$ rDNA hits & $328(0.21 \%)$ & $1100(.24 \%)$ \\
\hline Greengenes $16 \mathrm{~S}$ rDNA hits & $295(0.19 \%)$ & $912(0.20 \%)$ \\
\hline w/Func Prediction* & $33249(77.9 \%)$ & $93804(75.2 \%)$ \\
\hline COG* & 33997 (79.7\%) & $97053(77.8 \%)$ \\
\hline Pfam* & 34589 (81.0\%) & 99027 (79.4\%) \\
\hline TIGRfam* & $16117(37.8 \%)$ & 44040 (35.3\%) \\
\hline Genome Properties* & 3881 (9.1\%) & $10599(8.5 \%)$ \\
\hline Signalp* & 11125 (26.1\%) & $35780(28.7 \%)$ \\
\hline TransMb* & $8863(20.8 \%)$ & 26949 (21.6\%) \\
\hline MetaCyc* & 3694 (8.7\%) & $10815(8.7 \%)$ \\
\hline
\end{tabular}

* Indicates that these summary statistics were generated using the IMG/M-ER annotation system offered through the Joint Genome Institute [4] using the proxygene method [34].

metagenomic datasets to reveal phylotype differences between mammalian, avian, and invertebrate distal gut microbiomes. The distribution of bacterial phyla from swine feces appeared closest to that of the cow rumen and chicken cecum, sharing more similar proportions of Bacteroidetes, Firmicutes, Proteobacteria, and Actinobacteria (Figure 2). A statistical analysis comparing bacterial distribution between hosts revealed several significantly different bacterial groups. (Additional File 2 , Table S1 and S2). Human adult and infant distal gut microbiomes had significantly higher abundances of Actinobacteria $(\mathrm{p}<0.05)$ than did the swine microbiome (Additional File 2, Table S2). The fish gut microbiome was comprised mostly of Proteobacteria and Firmicutes, while the termite gut was dominated by Spirochetes. Interestingly, the swine fecal metagenome also harbored significantly more Spirochetes than many other hosts. (Additional File 2, Table S3).

Among the Bacteroidetes, Prevotella were significantly more abundant in the swine fecal metagenome when compared to all other gut metagenomes $(\mathrm{p}<0.05)$, with the exception of the cow rumen, while Bacteroides species were more abundant in chicken and human distal gut microbiomes (Figure 3). Additionally, Anaerovibrio and Treponema genera were exclusively found within the pig fecal metagenomes. Hierarchical clustering of phylotype distribution (genus-level) from each gut microbiome revealed that community structure of the swine fecal microbiome was significantly different $(\mathrm{p}<$ 0.05 ) from the other gut microbiomes (Figure 4A). Of all the microbiomes used in the comparative analysis, the swine metagenomes exhibited the highest resemblance to the cow rumen, displaying 59\% similarity at the genus level. Surprisingly, swine fecal community structure (genus-level) was less than $40 \%$ similar to any of the human fecal microbiomes used in this study.

\section{Diversity of swine gut microbiome}

In order to assess diversity of each gut metagenome, several statistical models were applied for measuring genotype richness, evenness, and coverage of rRNA gene hits against the RDP database. Overall, while coverage of the GS20 pig fecal metagenome was slightly lower than the FLX run (91\% vs 97\%), all diversity indices showed that both swine metagenomes had similar genotype diversity (Table 2). Swine fecal microbiomes appeared to have higher richness and lower evenness as compared to chicken, mouse, fish, and termite gut communities. This trend was further supported by a cumulative kdominance plot, as both swine k-dominance curves are less elevated than all other gut microbiomes (Additional File 1, Fig. S4). Rarefaction of the observed number of 
A.

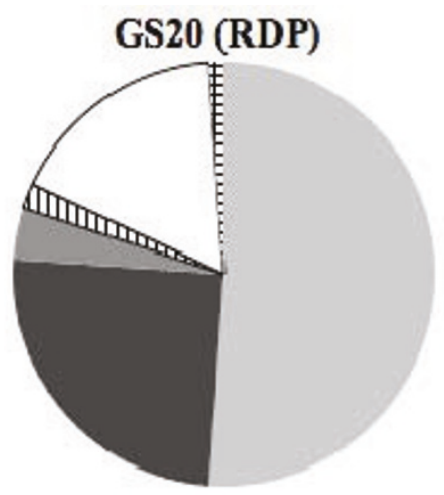

C.

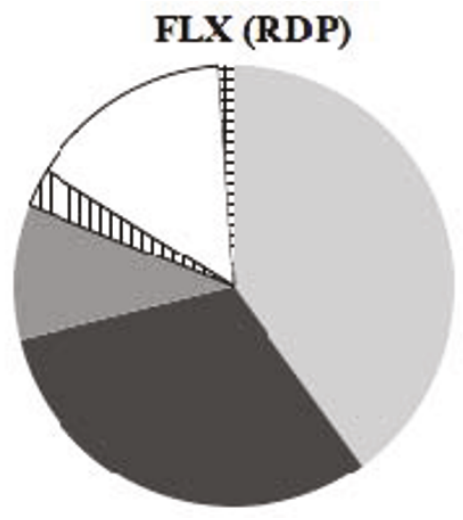

B.

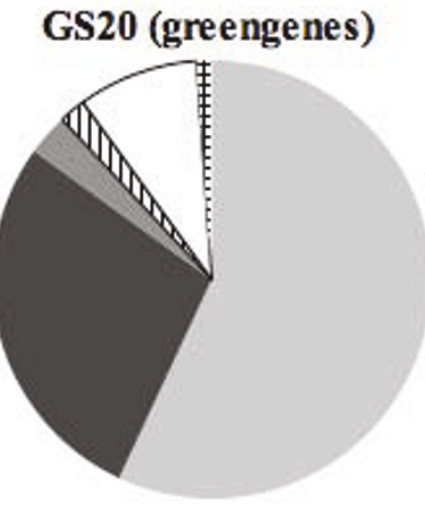

Firmicutes

Bacteroidetes

Proteobacteria

mSpirochaetes

口Unclassified Bacteria

\# Other Bacteria

D.

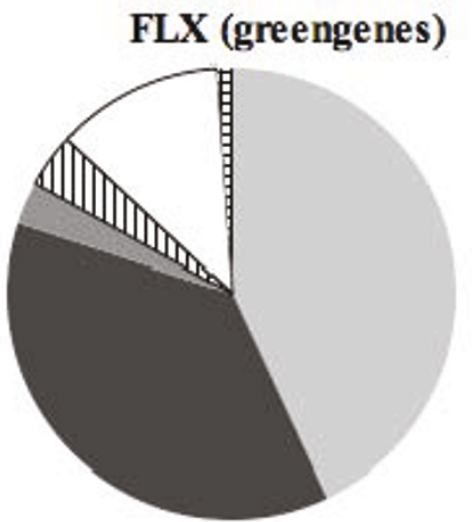

Figure 1 Taxonomic composition of bacterial phyla using 16S rRNA gene sequences retrieved from GS20 and FLX swine fecal metagenomes. Using the "Phylogenetic Analysis" tool within MG-RAST, the GS20 and FLX sequencing runs were searched against the RDP and greengenes databases using the BLASTn algorithm. The percent of sequences assigned to each of the bacterial phyla from the pig fecal GS20 (A and $B$ ) and FLX ( $C$ and D) metagenomes is shown. The e-value cutoff for 165 rRNA gene hits to RDP and greengenes databases was $1 \times 10^{-5}$ with a minimum alignment length of $50 \mathrm{bp}$.

OTUs (genus-level) indicated several of the individual human microbiomes were under-sampled (Additional File 1, Fig. S5), thus, we combined individual pig fecal, human infant, and human adult rRNA gene hits, and also performed diversity analyses on the total number of rRNA gene hits (Table 2). While the number of rRNA gene sequences in metagenome projects is low, comparison between available metagenomes showed that the human adult and pig microbiomes shared similar diversity patterns, and were more diverse than human infant microbiota.

\section{Functional classification of the swine gut metagenome}

To predict the metabolic potential within the swine fecal microbiome, both the MG-RAST and the IMG/M-ER annotation pipelines were used. The broad functional classifications of the swine fecal metagenomic reads were expected from previous metagenomic studies of the chicken cecum, cow rumen, human distal gut, and the termite gut. Similar proportions of broad level SEED subsystem classification were retrieved for both the GS20 and FLX swine fecal metagenomes (Additional File 1, Fig. S6). However, only $10 \%$ of sequences retrieved from the GS20 pig fecal metagenome were assigned to 574 subsystems, while more than $25 \%$ of all FLX reads were classified into 714 subsystems. This is compatible with the longer reads produced by the latter instrument, which allows for more robust gene predictions. When both pig fecal metagenomes were annotated using proxygenes within the JGI IMG/M ER pipeline, nearly one third of all GS20 and FLX pig fecal metagenomes were assigned to Pfams, and over $20 \%$ were assigned to COGs. This finding suggests that the proxygene method for gene-centric approaches to 

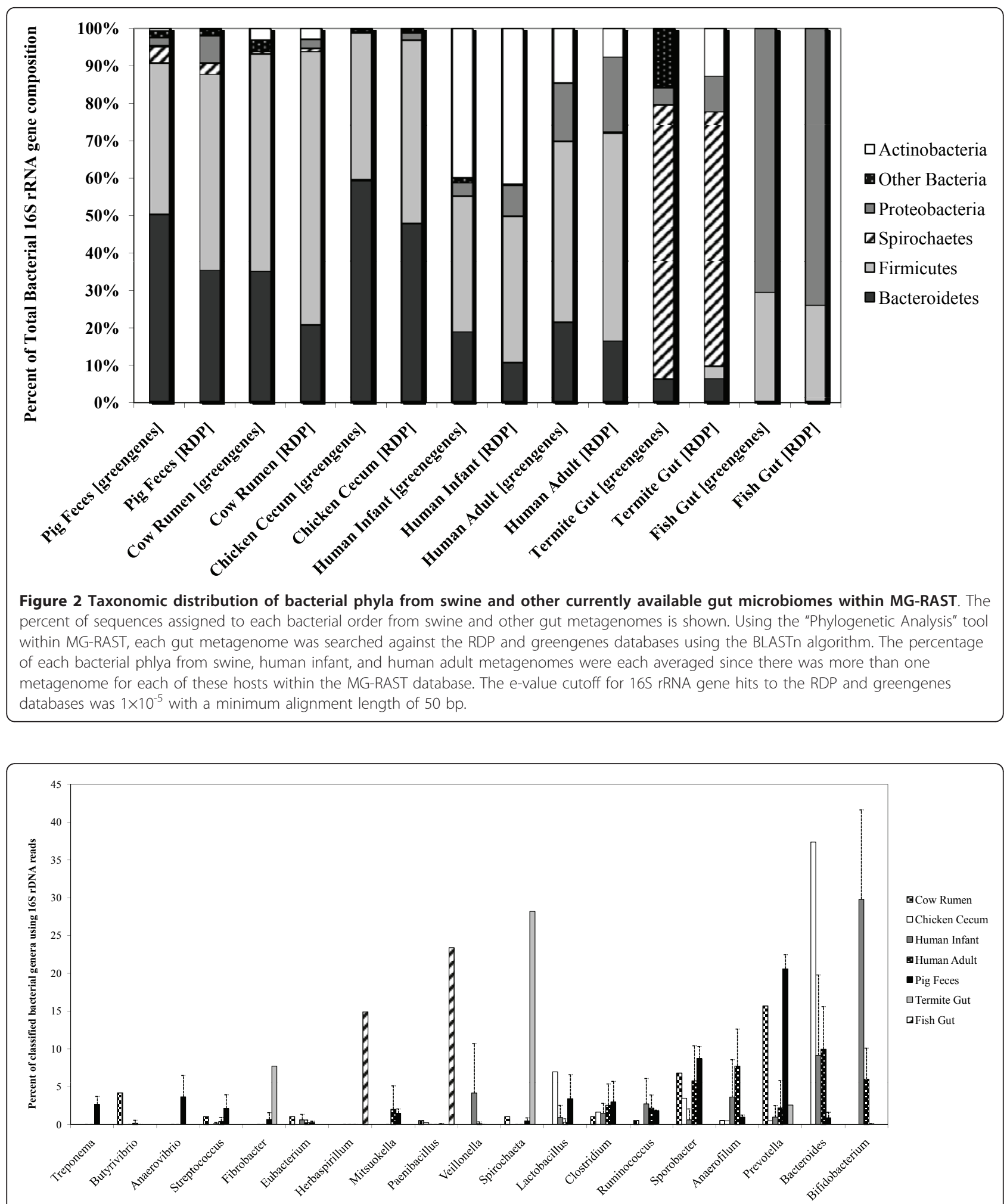

Figure 3 Taxonomic distribution of bacterial genera from swine and other currently available gut microbiomes within MG-RAST. The percent of sequences assigned to each bacterial order from swine and other gut metagenomes is shown. Using the "Phylogenetic Analysis" tool within MG-RAST, each gut metagenome was searched against the RDP and greengenes databases using the BLASTn algorithm. The percentage of each bacterial phlya from swine, human infant, and human adult metagenomes were each averaged since there was more than one metagenome for each of these hosts within the MG-RAST database. The e-value cutoff for 16S rRNA gene hits to the RDP and greengenes databases was $1 \times 10^{-5}$ with a minimum alignment length of $50 \mathrm{bp}$. 
A.

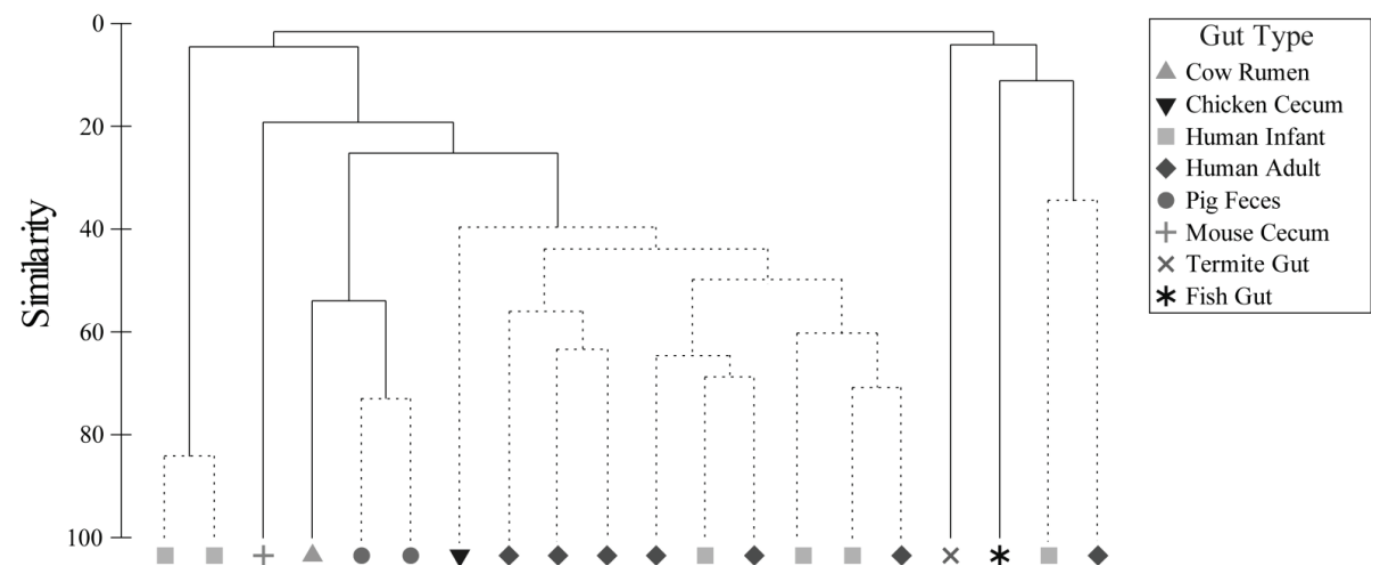

B.

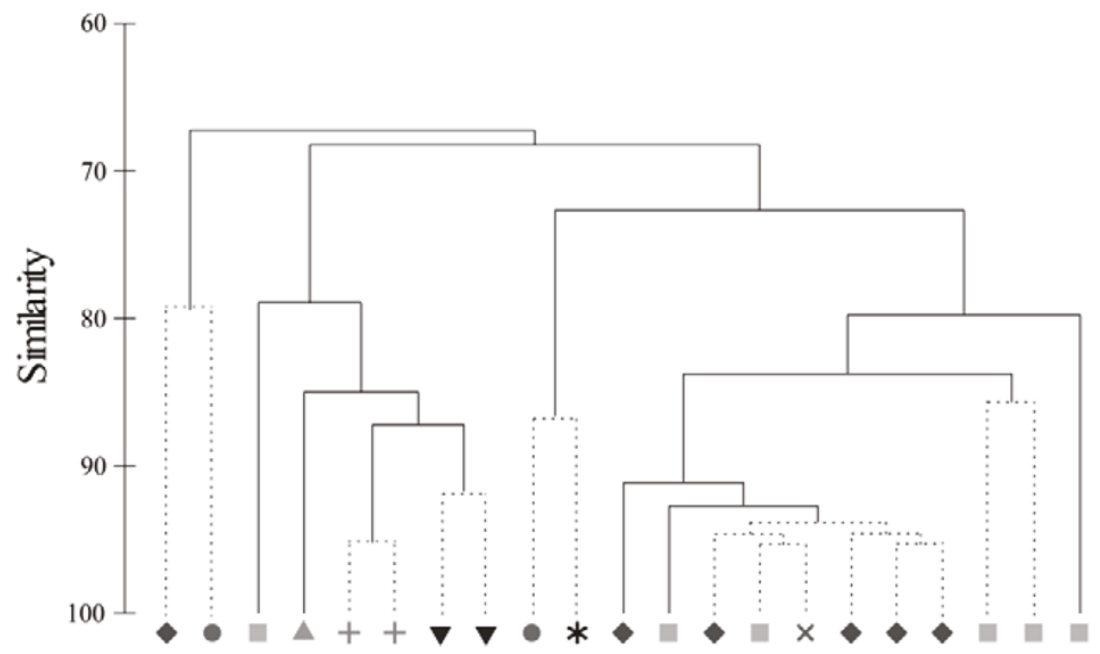

\begin{tabular}{|l|}
\hline \multicolumn{1}{|c|}{ Gut Type } \\
Cow Rumen \\
$\boldsymbol{\nabla}$ Chicken Cecum \\
Human Infant \\
Human Adult \\
Mouse Cecum \\
+ Pig Feces \\
$\times$ Fish Gut \\
* Termite Gut \\
\hline
\end{tabular}

Figure 4 Hierarchical clustering of gut metagenomes available within MG-RAST based on the taxonomic (A) and functional (B) composition. A matrix consisting of the number of reads assigned to the RDP database was generated using the "Phylogenetic Analysis" tool within MG-RAST, using the BLASTn algorithm. The e-value cutoff for $16 \mathrm{~S}$ rRNA gene hits to the RDP database was $1 \times 10^{-5}$ with a minimum alignment length of $50 \mathrm{bp}$. A matrix consisting of the number of reads assigned to SEED Subsytems from each gut metagenome was generated using the "Metabolic Analysis" tool within MG-RAST. The e-value cutoff for metagenomic sequence matches to this SEED Subsystem was $1 \times 10^{-5}$ with a minimum alignment length of $30 \mathrm{bp}$. Resemblance matrices were calculated using Bray-Curtis dissimilarities within PRIMER v6 software [38]. Clustering was performed using the complete linkage algorithm. Dotted branches denote that no statistical difference in similarity profiles could be identified for these respective nodes, using the SIMPROF test within PRMERv6 software.

metagenomic studies is more robust than the direct BLASTx assignment strategy. Diversity analyses of Subsystems, COGs, and Pfams retrieved from swine metagenomes and other gut metagenomes tested in this study, revealed that larger sequencing efforts generate significantly more functional classes (Additional File 2, Tables S4 \& S5). For example, an additional 150 Subsystems, 896 COGs, and 1271 Pfams were retrieved from the FLX run as compared to the GS20 metagenome, suggesting additional sequencing efforts for all gut microbiomes are necessary to cover the high functional diversity in gut environments.

Carbohydrate metabolism was the most abundant SEED subsystem (MG-RAST annotation pipeline) representing $13 \%$ of both swine fecal metagenomes (Additional File 1, Fig. S6). Genes associated with cell wall and capsule, stress, and virulence were also very abundant in both metagenomes. Approximately 16\% of annotated reads from swine fecal metagenomes were categorized within the clustering-based 
Table 2 Diversity analyses of the gut microbiomes using 16S rRNA gene sequences

\begin{tabular}{|c|c|c|c|c|c|c|c|c|c|}
\hline Metagenome & Sobs & Chao1 & ACE & Jackknife & Shannon & $\begin{array}{c}\text { Shannon (non- } \\
\text { parametric) }\end{array}$ & Simpson & boneh & coverage \\
\hline $\begin{array}{l}\text { Pig Feces } \\
\text { GS20 }\end{array}$ & 52 & $\begin{array}{c}77.09(61.24- \\
120.12)\end{array}$ & $\begin{array}{c}116.05(89.07- \\
162.68)\end{array}$ & $\begin{array}{c}76.88(62.74- \\
91.02)\end{array}$ & $\begin{array}{c}3.17(3.03- \\
3.32)\end{array}$ & 3.36 & $\begin{array}{c}0.07(0.05- \\
0.08)\end{array}$ & 10.34 & 0.91 \\
\hline Pig Feces FLX & 71 & $\begin{array}{c}113.86(86.42- \\
190.10)\end{array}$ & $\begin{array}{c}125.60(103.78- \\
161.95)\end{array}$ & $\begin{array}{c}119.78(92.49- \\
147.06)\end{array}$ & $\begin{array}{c}3.19(3.10- \\
3.29)\end{array}$ & 3.27 & $\begin{array}{c}0.08(0.07- \\
0.09)\end{array}$ & 5.84 & 0.97 \\
\hline Cow Rumen & 40 & $\begin{array}{c}63.00(48.33- \\
103.51)\end{array}$ & $\begin{array}{c}168.17(120.97- \\
242.89)\end{array}$ & $\begin{array}{c}63.63(49.92- \\
77.33)\end{array}$ & $\begin{array}{l}2.56(2.35- \\
2.77)\end{array}$ & 2.86 & $\begin{array}{c}0.15(0.11- \\
0.19)\end{array}$ & 10.58 & 0.88 \\
\hline $\begin{array}{l}\text { Chicken } \\
\text { Cecum }\end{array}$ & 37 & $\begin{array}{c}47.11(39.89- \\
72.43)\end{array}$ & $\begin{array}{c}68.02(52.45- \\
99.29)\end{array}$ & $\begin{array}{c}51.00(40.63- \\
61.37)\end{array}$ & $\begin{array}{l}2.25(2.11- \\
2.39)\end{array}$ & 2.36 & $\begin{array}{c}0.20(0.17- \\
0.23)\end{array}$ & 5.58 & 0.97 \\
\hline Human In-A & 20 & $\begin{array}{l}33.75(23.40- \\
\quad 75.55)\end{array}$ & $\begin{array}{c}62.23(41.01- \\
104.88)\end{array}$ & $\begin{array}{c}32.94(22.19- \\
43.70)\end{array}$ & $\begin{array}{l}2.52(2.25- \\
2.79)\end{array}$ & 2.84 & $\begin{array}{c}0.10(0.06- \\
0.15)\end{array}$ & 5.05 & 0.81 \\
\hline Human In-B & 10 & $\begin{array}{c}20.50(12.03- \\
64.19)\end{array}$ & $\begin{array}{c}27.79(13.32- \\
105.26)\end{array}$ & $\begin{array}{c}23.03(10.30- \\
35.76)\end{array}$ & $\begin{array}{c}0.84(0.50- \\
1.17)\end{array}$ & 1.15 & $\begin{array}{c}0.68(0.53- \\
0.82)\end{array}$ & 3.02 & 0.90 \\
\hline Human In-D & 26 & $\begin{array}{c}32.00(27.33- \\
53.10)\end{array}$ & $\begin{array}{c}34.06(28.41- \\
52.93)\end{array}$ & $\begin{array}{c}35.00(26.68- \\
43.32)\end{array}$ & $\begin{array}{l}2.97(2.80- \\
3.13)\end{array}$ & 3.16 & $\begin{array}{c}0.05(0.04- \\
0.07)\end{array}$ & 4.95 & 0.90 \\
\hline Human In-E & 18 & $\begin{array}{c}22.20(18.79- \\
40.34)\end{array}$ & $\begin{array}{c}26.41(20.24- \\
49.62)\end{array}$ & $\begin{array}{c}25.00(17.67- \\
32.33)\end{array}$ & $\begin{array}{l}1.11(0.88- \\
1.34)\end{array}$ & 1.26 & $\begin{array}{l}0.60(0.51- \\
0.69)\end{array}$ & 3.72 & 0.96 \\
\hline Human In-M & 26 & $\begin{array}{c}46.00(32.02- \\
92.48)\end{array}$ & $\begin{array}{c}80.76(54.86- \\
129.91)\end{array}$ & $\begin{array}{c}43.95(31.51- \\
56.39)\end{array}$ & $\begin{array}{l}2.97(2.72- \\
3.22)\end{array}$ & 3.42 & $\begin{array}{c}0.05(0.02- \\
0.08)\end{array}$ & 7.34 & 0.69 \\
\hline Human In-R & 21 & $\begin{array}{c}23.50(21.41- \\
36.27)\end{array}$ & $\begin{array}{c}26.77(22.44- \\
44.13)\end{array}$ & $\begin{array}{c}27.00(20.21- \\
33.79)\end{array}$ & $\begin{array}{l}2.57(2.38- \\
2.76)\end{array}$ & 2.72 & $\begin{array}{c}0.10(0.07- \\
0.13)\end{array}$ & 2.83 & 0.87 \\
\hline Human F1-S & 22 & $\begin{array}{l}31.00(24.00- \\
62.45)\end{array}$ & $\begin{array}{l}39.21(29.33- \\
62.40)\end{array}$ & $\begin{array}{c}31.00(22.68- \\
39.32)\end{array}$ & $\begin{array}{l}2.68(2.49- \\
2.87)\end{array}$ & 2.85 & $\begin{array}{l}0.08(0.06- \\
0.10)\end{array}$ & 4.30 & 0.90 \\
\hline Human F1-T & 37 & $\begin{array}{c}64.14(46.04- \\
118.51)\end{array}$ & $\begin{array}{c}109.84(79.72- \\
161.17)\end{array}$ & $\begin{array}{c}66.22(47.95- \\
84.48)\end{array}$ & $\begin{array}{l}3.05(2.83- \\
3.26)\end{array}$ & 3.36 & $\begin{array}{c}0.07(0.04- \\
0.10)\end{array}$ & 9.39 & 0.82 \\
\hline Human F1-U & 17 & $\begin{array}{c}20.75(17.64- \\
39.02)\end{array}$ & $\begin{array}{c}21.96(18.14- \\
38.53)\end{array}$ & $\begin{array}{c}23.00(16.21- \\
29.79)\end{array}$ & $\begin{array}{c}2.30(2.04- \\
2.56)\end{array}$ & 2.49 & $\begin{array}{c}0.15(0.08- \\
0.21)\end{array}$ & 3.22 & 0.91 \\
\hline Human F2-V & 37 & $\begin{array}{c}46.10(39.59- \\
68.96)\end{array}$ & $\begin{array}{l}48.59(41.00- \\
70.52)\end{array}$ & $\begin{array}{c}51.00(40.63- \\
61.37)\end{array}$ & $\begin{array}{c}3.07(2.89- \\
3.26)\end{array}$ & 3.29 & $\begin{array}{c}0.07(0.05- \\
0.09)\end{array}$ & 7.64 & 0.87 \\
\hline Human F2-W & 25 & $\begin{array}{l}36.00(27.88- \\
66.94)\end{array}$ & $\begin{array}{l}55.50(39.11- \\
90.92)\end{array}$ & $\begin{array}{c}37.00(27.40- \\
46.60)\end{array}$ & $\begin{array}{l}2.72(2.50- \\
2.93)\end{array}$ & 2.96 & $\begin{array}{c}0.08(0.06- \\
0.11)\end{array}$ & 5.85 & 0.86 \\
\hline Human F2-X & 19 & $\begin{array}{c}21.00(19.29- \\
32.96)\end{array}$ & $\begin{array}{c}22.80(19.83- \\
36.32)\end{array}$ & $\begin{array}{c}24.00(17.80- \\
30.20)\end{array}$ & $\begin{array}{l}2.57(2.38- \\
2.76)\end{array}$ & 2.72 & $\begin{array}{c}0.09(0.06- \\
0.12)\end{array}$ & 3.06 & 0.94 \\
\hline Human F2-Y & 27 & $\begin{array}{c}40.20(30.44- \\
77.60)\end{array}$ & $\begin{array}{c}41.54(31.66- \\
72.36)\end{array}$ & $\begin{array}{c}39.78(29.54- \\
50.01)\end{array}$ & $\begin{array}{c}2.87(2.67- \\
3.08)\end{array}$ & 3.10 & $\begin{array}{c}0.07(0.05- \\
0.09)\end{array}$ & 5.82 & 0.87 \\
\hline Mouse Cecum & 14 & $\begin{array}{c}36.50(19.23- \\
110.77)\end{array}$ & $\begin{array}{l}41.22(20.35- \\
130.67)\end{array}$ & $\begin{array}{c}39.09(19.22- \\
58.95)\end{array}$ & $\begin{array}{l}2.18(1.78- \\
2.58)\end{array}$ & 2.69 & $\begin{array}{c}0.15(0.04- \\
0.25)\end{array}$ & 4.13 & 0.67 \\
\hline Termite Gut & 13 & $\begin{array}{l}27.00(15.92- \\
80.11)\end{array}$ & $\begin{array}{c}30.75(16.84- \\
95.03)\end{array}$ & $\begin{array}{c}29.19(14.56- \\
43.82)\end{array}$ & $\begin{array}{c}2.05(1.72- \\
2.38)\end{array}$ & 2.38 & $\begin{array}{c}0.16(0.09- \\
0.23)\end{array}$ & 3.39 & 0.79 \\
\hline Fish gut & 14 & $\begin{array}{c}19.00(14.86- \\
42.91)\end{array}$ & $\begin{array}{c}20.45(15.44- \\
42.93)\end{array}$ & $\begin{array}{c}20.00(13.21- \\
26.79)\end{array}$ & $\begin{array}{l}2.29(2.05- \\
2.54)\end{array}$ & 2.50 & $\begin{array}{c}0.11(0.07- \\
0.15)\end{array}$ & 3.71 & 0.87 \\
\hline $\begin{array}{l}\text { Pig Feces } \\
\text { Total }\end{array}$ & 91 & $\begin{array}{c}127.25(105.56- \\
181.27)\end{array}$ & $\begin{array}{c}184.42(150.70- \\
237.20)\end{array}$ & $\begin{array}{c}127.57(108.75- \\
146.39)\end{array}$ & $\begin{array}{c}3.15(3.11- \\
3.20)\end{array}$ & 3.19 & $\begin{array}{c}0.06(0.06- \\
0.07)\end{array}$ & 0.34 & 0.99 \\
\hline $\begin{array}{c}\text { Human Infant } \\
\text { Total }\end{array}$ & 59 & $\begin{array}{c}80.00(66.47- \\
118.05)\end{array}$ & $\begin{array}{c}83.37(69.43- \\
115.92)\end{array}$ & $\begin{array}{c}82.03(68.30- \\
95.75)\end{array}$ & $\begin{array}{c}2.66(2.52- \\
2.79)\end{array}$ & 2.78 & $\begin{array}{c}0.17(0.14- \\
0.20)\end{array}$ & 1.25 & 0.96 \\
\hline $\begin{array}{c}\text { Human Adult } \\
\text { Total }\end{array}$ & 72 & $\begin{array}{c}89.00(77.34- \\
126.16)\end{array}$ & $\begin{array}{c}85.74(77.28- \\
107.71)\end{array}$ & $\begin{array}{c}89.60(77.72- \\
101.48)\end{array}$ & $\begin{array}{c}3.35(3.30- \\
3.40)\end{array}$ & 3.39 & $\begin{array}{c}0.05(0.04- \\
0.05)\end{array}$ & 0.37 & 0.99 \\
\hline
\end{tabular}

subsystems, most of which have unknown or putative functions. Additionally, $75 \%$ to $90 \%$ of metagenomic reads were not assigned to subsystems, suggesting the need for improved binning and coding region prediction algorithms to annotate these unknown sequences.

To improve the meaning of metagenomic functional analysis, we applied statistical methods to compare the 29 broad level functional subsystems that are more or less represented in the different microbiomes. As was expected, all gut metagenomes were dominated by carbohydrate metabolism subsystems with amino acid, protein, cell wall and capsule, and virulence subsystems represented in relatively high abundance as well. Protein metabolism and amino acid subsystems were significantly more abundant in chicken, pig, and cow gut metagenomes (Additional File 1, Fig. S7). Additionally, the termite, fish, and pig gut had a higher proportion of 
reads classified to the chemotaxis and motility subsystems as compared to other gut metagenomes.

\section{Comparative gut metagenomics}

In this study, we examined the functional similarity of the Yorkshire pig fecal metagenome by comparing it to currently available metagenomic projects. Hierarchical clustering of functional profiles derived from gut metagenomes available in the MG-RAST database revealed that the GS20 and FLX swine fecal datasets shared approximately $70 \%$ similarity to other human metagenomes (Figure 4B). This analysis also showed the swine gut metagenome clustered more closely with chicken cecal and cow rumen metagenomes than to the human gut metagenomes (Figure 4B).

We further investigated subsystems associated with specialized cell wall and capsule enzymes, DNA recombination, and prophage genes since they were very abundant in the swine fecal metagenome (Additional File 1, Fig. S8). Within the DNA recombination and prophage subsystem, the swine fecal metagenome was enriched for RstA phage-related replication proteins, terminases, and portal proteins. Additionally, more than 30 metagenomic contigs (i.e., > $500 \mathrm{bp}$ ) shared high homology to unknown phage proteins. For proteins involved in the cell wall and capsule subsystem, unknown glycosyl transferases, a phosphoglucosamine mutase, and a phosphotransferase were over abundant in the swine metagenome (Table 3). N-acetyl glucosamine-specific PTS system, proteins involved in mannose uptake, and novel capsular polysaccharide synthesis enzymes were exclusively found within the swine fecal metagenome. Hierarchical clustering of all genes retrieved from the cell wall and capsule functional subsystem for each gut microbiome revealed that swine fecal cell wall/capsule profiles were greater than $60 \%$ similar to those of the cow rumen. Additionally, cell wall and capsule profiles in the swine samples were more similar to termite gut than the human gut (Additional File 1, Fig. S9). When carbohydrate subsystems were compared across gut microbiomes, maltose and maltodextrin utilization were the most abundant carbohydrate subsystem in the swine, termite, and cow rumen. Analysis of carbohydrate metabolism using the SEED subsystem approach, revealed several proteins unique to the swine gut metagenome such as an outer surface protein part of the cellobiose operon, a beta-glucoside-specific IIA component and a cellobiose-specific IIC component of the PTS system, and a protein similar CDP-glucose 4,6-dehydratase.

Two-way hierarchical clustering of COGs retrieved from swine, human, termite, and mouse gut microbiomes revealed several suites of gene families unique to the swine distal gut (Figure 5). Additionally, the swine fecal FLX run yielded a pool COGs unique to the FLX run, suggesting the deeper level of sequencing uncovered a larger proportion of functional diversity. Interestingly, this analysis unveiled a large collection of COGs unique to the swine fecal metagenome.

\section{Discussion}

The primary goal of this study was to characterize the functional content of the swine fecal microbiome. We also compared the pig distal gut samples to other currently available gut metagenomes, as a method for revealing potential differences in gut microbial systems. The comparative metagenomic approach used in this

Table 3 List of cell wall and capsule SEED subsystem functions overabundant in swine fecal metagenome

\begin{tabular}{|c|c|c|c|c|c|c|c|}
\hline & $\begin{array}{l}\text { Pig } \\
\text { Feces }\end{array}$ & $\begin{array}{l}\text { Human } \\
\text { Adult }\end{array}$ & $\begin{array}{l}\text { Human } \\
\text { Infant }\end{array}$ & $\begin{array}{l}\text { Cow } \\
\text { Rumen }\end{array}$ & $\begin{array}{l}\text { Termite } \\
\text { Gut }\end{array}$ & $\begin{array}{l}\text { Mouse } \\
\text { Cecum }\end{array}$ & $\begin{array}{l}\text { Fish } \\
\text { gut }\end{array}$ \\
\hline $\begin{array}{l}\text { putative glycosyltransferase - possibly involved in cell wall localization and } \\
\text { side chain formation of rhamnose-glucose polysaccharide }\end{array}$ & 112 & 9 & 10 & 10 & 0 & 1 & 0 \\
\hline Phosphoglucosamine mutase (EC 5.4.2.10) & 97 & 18 & 9 & 0 & 20 & 0 & 1 \\
\hline COG3178: Predicted phosphotransferase related to Ser/Thr protein kinases & 66 & 10 & 6 & 4 & 5 & 2 & 1 \\
\hline 3-deoxy-D-manno-octulosonate 8-phosphate phosphatase (EC 3.1.3.45) & 27 & 10 & 9 & 2 & 0 & 1 & 3 \\
\hline O-antigen export system, permease protein & 23 & 3 & 2 & 4 & 0 & 0 & 1 \\
\hline Glutamine synthetase, clostridia type (EC 6.3.1.2) & 21 & 4 & 1 & 3 & 0 & 0 & 0 \\
\hline D-glycero-D-manno-heptose 1-phosphate guanosyltransferase & 20 & 7 & 6 & 1 & 0 & 5 & 0 \\
\hline UDP-glucose 4-epimerase (EC 5.1.3.2) & 14 & 1 & 2 & 0 & 9 & 1 & 1 \\
\hline Capsular polysaccharide synthesis enzyme Cap8D & 9 & 0 & 1 & 1 & 0 & 0 & 0 \\
\hline D-alanine-D-alanine ligase B (EC 6.3.2.4) & 8 & 0 & 0 & 0 & 0 & 0 & 0 \\
\hline PTS system, N-acetylglucosamine-specific IIB component (EC 2.7.1.69) & 7 & 0 & 0 & 0 & 0 & 0 & 0 \\
\hline Mannose-1-phosphate guanylyltransferase (GDP) (EC 2.7.7.22) & 5 & 0 & 0 & 0 & 0 & 0 & 0 \\
\hline 2-Keto-3-deoxy-D-manno-octulosonate-8-phosphate synthase (EC 2.5.1.55) & 3 & 0 & 0 & 0 & 0 & 0 & 0 \\
\hline capsular polysaccharide biosynthesis protein, putative & 3 & 0 & 0 & 0 & 0 & 0 & 0 \\
\hline Capsular polysaccharide synthesis enzyme Cap8L & 3 & 0 & 0 & 0 & 0 & 0 & 0 \\
\hline
\end{tabular}




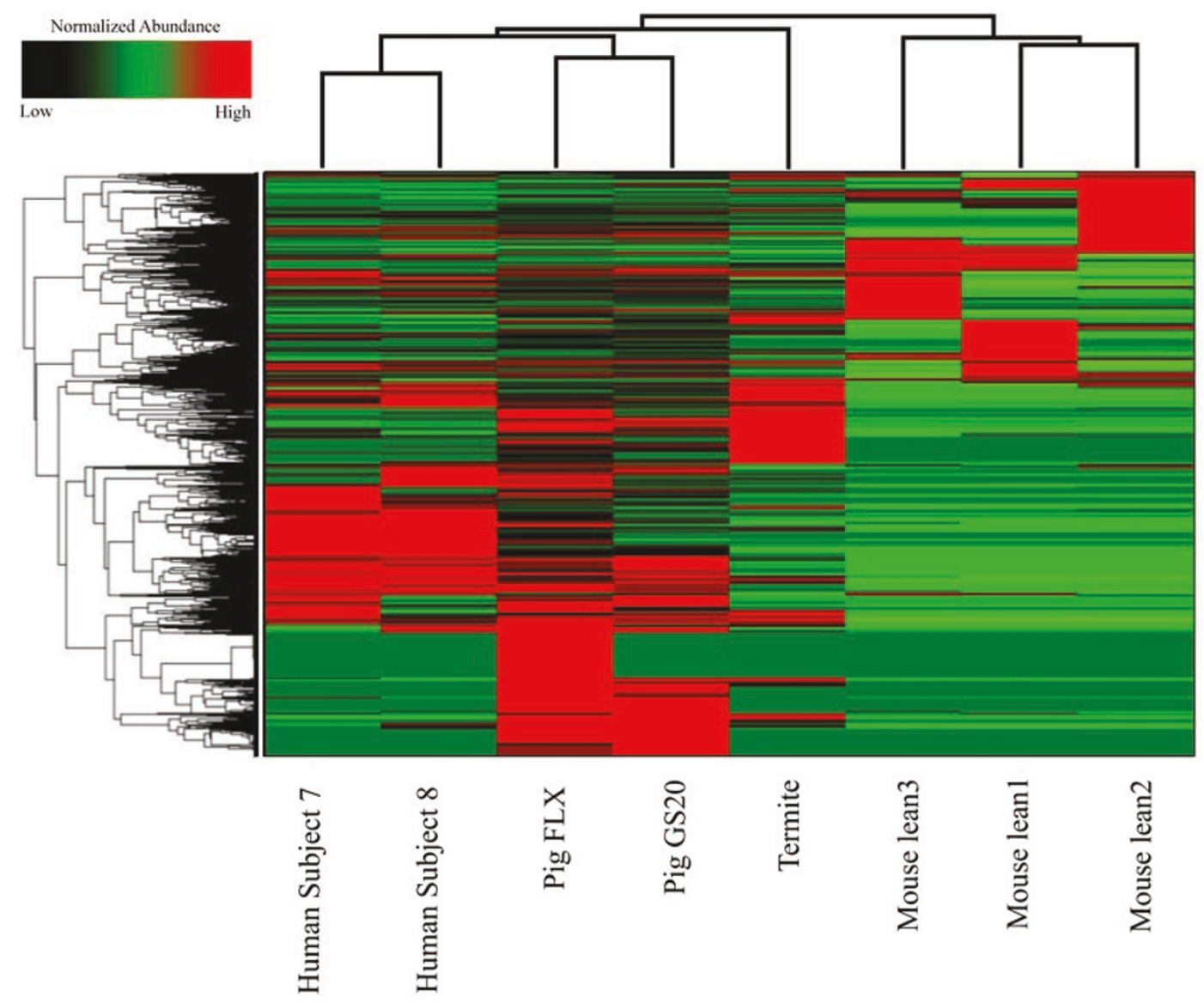

Figure 5 Two-way hierarchical clustering of functional gene groups from swine and other currently available gut metagenomes within JGI's IMG/M database. Hierarchical clustering was performed using a matrix of the number of reads assigned to COGs from each gut metagenome, which was generated using the "Compare Genomes" tool in IMG/M ER. COGs less abundant in a given metagenome are shown in black/darkgreen, while more abundant COGs are shown in red.

study identified unique and/or overabundant taxonomic and functional elements within the swine distal gut. It also appears that the genes associated with the variable portion of gut microbiomes cluster by host environment with surprising hierarchical trends. Thus, our findings suggest that while a majority of metagenomic reads were associated with a relatively conserved core microbiome, the variable microbiome carries out many unique functions [8]. The data also suggest that taxonomically diverse gut organisms maintain a conserved core set of genes, although it should be noted that the variable microbiome is more abundant than previously anticipated. For example, of the 160 functional SEED Subsystems, DNA repair/recombination subsystems were amongst the most abundant functions within all gut microbiomes. Since the frequency of a gene encoding a particular metabolic function is usually related to its relative importance in an environment [8], transferable elements are likely to be very important in shaping microbiome composition and diversity in gastrointestinal environments. When comparing prophage and transposon genes from each gut microbiome, the pig distal microbiome examined in this study harbored an abundant and diverse array of horizontal gene transfer mechanisms. When putative transposases for all available gut metagenomes were retrieved using the IMG/M annotation pipeline, the swine fecal metagenome harbored the most diverse transposase profiles (i.e., 26 different transposase families; Additional File 1, Fig. S10). The potential importance of transposable elements was further supported by the fact that $42 \%$ of large contigs (> $500 \mathrm{bp}$ ) assembled from all pig fecal metagenomic contained sequences that matched putative transposases (Table 4). Additionally, $24 \%$ of all large contigs matched to proteins associated with antibiotic resistance mechanisms. These results suggest that lateral gene transfer and 
Table 4 Summary of BLASTX results of pig fecal assembled contigs

\begin{tabular}{|c|c|c|c|c|c|c|c|}
\hline $\begin{array}{l}\text { Contig } \\
\text { Name }\end{array}$ & $\begin{array}{l}\text { Contig } \\
\text { Length }\end{array}$ & $\begin{array}{l}\text { Number of } \\
\text { Reads }\end{array}$ & Predicted Protein & Organism & $\begin{array}{l}\text { Accession } \\
\text { Number }\end{array}$ & E-value & $\begin{array}{l}\text { Percent } \\
\text { Identity }\end{array}$ \\
\hline Contig09884 & 1444 & 159 & hypothetical protein & Bacteroides fragilis & BAA95637 & 0 & $99 \%$ \\
\hline Contig00095 & 646 & 22 & $\begin{array}{c}\text { tetracycline resistant protein } \\
\text { TetQ }\end{array}$ & Bacteroides sp. D1 & ZP 04543830 & $2.00 \mathrm{E}-111$ & $99 \%$ \\
\hline Contig01271 & 812 & 22 & $\begin{array}{l}\text { tetracycline resistance } \\
\text { protein }\end{array}$ & Prevotella intermedia & AAB51122 & $3.00 \mathrm{E}-102$ & $98 \%$ \\
\hline Contig01956 & 731 & 17 & macrolide-efflux protein & Faecalibacterium prausnitzii A2-165 & ZP 05613628 & $3.00 \mathrm{E}-85$ & $99 \%$ \\
\hline Contig01189 & 549 & 14 & macrolide-efflux protein & Bacteroides finegoldii DSM 17565 & ZP 05859238 & $8.00 \mathrm{E}-83$ & $98 \%$ \\
\hline Contig00070 & 603 & 11 & $\begin{array}{l}\text { rRNA (guanine-N1- } \\
\text { )-methyltransferase }\end{array}$ & Faecalibacterium prausnitzii A2-165 & ZP 05614052 & $2.00 \mathrm{E}-81$ & $100 \%$ \\
\hline Contig07794 & 846 & 27 & putative transposase & Bacteroides fragilis & AAA22911 & 4.00E-81 & $98 \%$ \\
\hline Contig03360 & 671 & 10 & $\begin{array}{l}\text { ABC transporter, ATP- } \\
\text { binding protein }\end{array}$ & $\begin{array}{l}\text { Bacillus thuringiensis serovar } \\
\text { pondicheriensis BGSC 4BA1 }\end{array}$ & ZP 04090641 & $8.00 \mathrm{E}-77$ & $77 \%$ \\
\hline Contig09748 & 650 & 13 & $\begin{array}{l}\text { hypothetical protein } \\
\text { PRABACTJOHN } 03572\end{array}$ & Parabacteroides johnsonii DSM 18315 & ZP 03477882 & $9.00 \mathrm{E}-71$ & $77 \%$ \\
\hline Contig00180 & 846 & 26 & macrolide-efflux protein & Faecalibacterium prausnitzii A2-165 & ZP 05613628 & $6.00 \mathrm{E}-67$ & $90 \%$ \\
\hline Contig00608 & 527 & 7 & ISPg3, transposase & Prevotella tannerae ATCC 51259 & ZP 05734821 & 1.00E-59 & $67 \%$ \\
\hline Contig04843 & 578 & 7 & $\begin{array}{l}\text { hypothetical protein } \\
\text { COPEUT } 02459\end{array}$ & Coprococcus eutactus ATCC 27759 & ZP 02207638 & $2.00 \mathrm{E}-57$ & $88 \%$ \\
\hline Contig00340 & 847 & 24 & $\begin{array}{l}\text { conserved hypothetical } \\
\text { protein }\end{array}$ & Bacteroides sp. 43 47FAA & ZP 05257903 & $6.00 \mathrm{E}-56$ & $72 \%$ \\
\hline Contig02245 & 616 & 7 & putative transposase & $\begin{array}{l}\text { Bacteroides thetaiotaomicron VPI- } \\
5482\end{array}$ & NP 809147 & $3.00 \mathrm{E}-52$ & $62 \%$ \\
\hline Contig09776 & 531 & 9 & resolvase, $\mathrm{N}$ domain protein & Faecalibacterium prausnitzii A2-165 & ZP 05613620 & $5.00 \mathrm{E}-41$ & $100 \%$ \\
\hline Contig02310 & 557 & 11 & $\begin{array}{l}\text { replication initiator protein } \\
\text { A }\end{array}$ & Faecalibacterium prausnitzii A2-165 & ZP 05613624 & $1.00 \mathrm{E}-38$ & $100 \%$ \\
\hline Contig02075 & 524 & 9 & Transposase & Bacteroides fragilis 3112 & ZP 05284372 & 7.00E-38 & $92 \%$ \\
\hline Contig02837 & 529 & 7 & $\begin{array}{l}\text { hypothetical protein } \\
\text { CLOSS21 } 01510\end{array}$ & Clostridium sp. SS2/1 & ZP 02439046 & $6.00 \mathrm{E}-37$ & $67 \%$ \\
\hline Contig09732 & 632 & 11 & $\begin{array}{l}\text { hypothetical protein } \\
\text { BACCOP } 00975\end{array}$ & Bacteroides coprocola DSM 17136 & ZP 03009123 & $1.00 \mathrm{E}-35$ & $62 \%$ \\
\hline Contig09862 & 574 & 16 & $\begin{array}{l}\text { conserved hypothetical } \\
\text { protein }\end{array}$ & Oxalobacter formigenes HOxBLS & ZP 04576182 & $1.00 \mathrm{E}-34$ & $100 \%$ \\
\hline Contig00069 & 897 & 21 & regulatory protein & $\begin{array}{c}\text { Sphingobacterium spiritivorum ATCC } \\
33300\end{array}$ & ZP 03965851 & 4.00E-29 & $43 \%$ \\
\hline Contig00129 & 529 & 9 & transposase, putative & Bacteroides sp. 217 & ZP 05288481 & $8.00 \mathrm{E}-26$ & $75 \%$ \\
\hline Contig00130 & 674 & 11 & $\begin{array}{l}\text { hypothetical protein } \\
\text { BACCOP } 00975\end{array}$ & Bacteroides coprocola DSM 17136 & ZP 03009123 & $6.00 \mathrm{E}-24$ & $43 \%$ \\
\hline Contig09924 & 1355 & 55 & $\begin{array}{l}\text { conserved hypothetical } \\
\text { protein }\end{array}$ & $\begin{array}{l}\text { Magnetospirillum gryphiswaldense } \\
\text { MSR-1 }\end{array}$ & CAJ30045 & $2.00 \mathrm{E}-23$ & $45 \%$ \\
\hline Contig00140 & 552 & 13 & ISPg7, transposase & Cyanothece sp. PCC 8802 & $\begin{array}{c}\text { YP } \\
003135760\end{array}$ & $5.00 \mathrm{E}-23$ & $44 \%$ \\
\hline Contig00572 & 675 & 16 & transposase, putative & Bacteroides sp. 217 & ZP 05288481 & $2.00 \mathrm{E}-21$ & $57 \%$ \\
\hline Contig09792 & 556 & 9 & $\begin{array}{c}\text { hypothetical protein ALIPUT } \\
01364\end{array}$ & Alistipes putredinis DSM 17216 & ZP 02425220 & $2.00 \mathrm{E}-16$ & $67 \%$ \\
\hline Contig09902 & 528 & 14 & putative transposase & Lentisphaera araneosa HTCC2155 & ZP 01873850 & $2.00 \mathrm{E}-12$ & $63 \%$ \\
\hline Contig09796 & 867 & 17 & $\begin{array}{l}\text { hypothetical protein } \\
\text { CLONEX } 03424\end{array}$ & Clostridium nexile DSM 1787 & ZP 03291203 & $3.00 \mathrm{E}-07$ & $35 \%$ \\
\hline Contig01049 & 548 & 5 & $\begin{array}{l}\text { No significant similarity } \\
\text { found }\end{array}$ & - & - & - & - \\
\hline Contig04775 & 565 & 4 & $\begin{array}{l}\text { No significant similarity } \\
\text { found }\end{array}$ & - & - & - & - \\
\hline Contig09740 & 531 & 7 & $\begin{array}{l}\text { No significant similarity } \\
\text { found }\end{array}$ & - & - & - & - \\
\hline Contig09927 & 656 & 29 & $\begin{array}{l}\text { No significant similarity } \\
\text { found }\end{array}$ & - & - & - & - \\
\hline
\end{tabular}


mobile elements allow gut microbial populations to perpetually change their cell surface for sensing their environment and collecting nutrient resources present in the distal intestine [2].

Interestingly, a majority of these transposable elements belonged to the Bacteroidetes genomes. These genetic elements have been shown to aid in the adaptation of this diverse group of bacteria to the distal gut environments [2]. Many of the genetic features unique to the swine fecal metagenome encoded cell surface features of different Bacteroidetes populations, suggesting the adaptation of Bacteroidetes populations to distinct niches within the swine distal gut microbiome. While the precise role of diet, antibiotic usage, and genetics on shaping the ecology of the distal pig gut will require further study, it should be noted that industrialization of the swine industry has lead to the frequent use antibiotics to supplement the pig diet to maintain and increase meat production.

Studying the swine distal gut metagenome also shed light on the diversity and high occurrence of antibiotic resistance mechanisms employed by the microbiome (Additional File 1, Fig. S11). Antibiotics are widely used as additives in food or water within swine feeding operations to prevent and treat animal disease and to promote animal growth [19]. Seepage and runoff of swine waste into both surface and groundwater with antibiotics and antibiotic-resistant bacteria poses a significant threat to public health. Nearly $6 \%$ of all assigned metagenomic reads retrieved from both swine fecal metagenomes were involved in antibiotic resistance mechanisms. Interestingly, tetracycline resistance was the most abundant class of virulence subsystems within the swine fecal metagenome, which may be explained by the fact that this antibiotic class was used in the diet supplied to the animals associated with this study. This antibiotic class is reported as comprising nearly half of the total amount of antibiotics used in commercial swine operations [20].

Resistance to fluoroquinolones was also well represented in the swine fecal metagenome, and may be explained by the increase of its non-therapeutic use within pig feed. While early studies indicated there was a low risk of fluoroquinolone resistance, recent studies are showing the use of fluoroquinolones is among the most important factors associated with finding resistant E. coli and Campylobacter in animal operations [21]. Interestingly, there was no history of fluoroquinolone use on the swine farm from which these samples were collected. Fluoroquinolone resistance has been found on farms with no history of fluoroquinolone use, suggesting that resistant organisms, such as Campylobacter have the ability to spread between pig farms. Genes with high sequence similarity to methicillin-resistant Staphylococcus subsystem were also retrieved in this study. This finding is important considering MRSA carriage has been elevated in swine and exposed farmers and veterinarians [22], suggesting that MRSA infection is a significant risk in swine farm resident and worker cohorts.

More than $12 \%$ of virulence subsystems identified in the pig fecal metagenome were classified as multi-drug resistance mechanisms, suggesting the pig gut could be a hot-spot for multiple-antibiotic resistant bacteria. One subsystem, the MexA-MexB-OprM multiple drug efflux pump was found exclusively in the swine fecal metagenome. This antibiotic resistance mechanism has been detected only in Pseudomonas aeruginosa strains known to carry resistance in cystic fibrosis patients [23] and has not been previously described in distal gut environments. Additionally, more than $10 \%$ of virulence-associated sequences were assigned to yet-to-be-described virulence subsystems, suggesting that unknown virulence mechanisms are at work within the distal gut. Altogether, the high abundance of metagenomic sequences assigned to known and unknown antibiotic resistance subsystems suggests that functional metagenomics is an adequate tool for assessing the prevalence of antibiotic resistance within high cell density environments.

Pair-wise comparisons of each gut metagenome (MGRAST SEED database) with the swine gut revealed 15 SEED subsystems that were significantly different in abundance for the swine fecal metagenome (Figure 6 and Additional File 1, Fig. S12). Two subsystems in particular were statistically significantly overabundant in the swine gut metagenome, as compared to all other gut metagenomes: the UDP-N-acetylmuramate from fructose-6-phosphate biosynthesis and folate biosynthesis. UDP-N-acetylmuramate is a peptidoglycan-derived muropeptide that as a group are considered to be potential virulence factors of several gut pathogens [24] specifically involved in biofilm colonization. Higher abundances of genes related to folate biosynthesis may be a direct result of supplemental amounts of folic acid in swine feedstuff or an increased production by the swine microbial consortia [25]. The impacts of food additives, such as folic acid, on the microbial ecology of the swine gut warrants further study.

Comparative metagenomics of proteins involved in the cell wall and capsule subsystems revealed several unique glycosyl transferases and carbohydrate uptake systems. This unique pool of glycosyl transferases may provide a capacity for diversification of surface polysaccharide structures helping shape the genetic functional potential of this gut ecosystem. For example, the acquisition of new types of carbohydrate-binding proteins, transporters, and degradation enzymes through horizontal gene transfer may allow for the utilization of a wider array of substrates that may be utilized for energy harvesting [2]. 


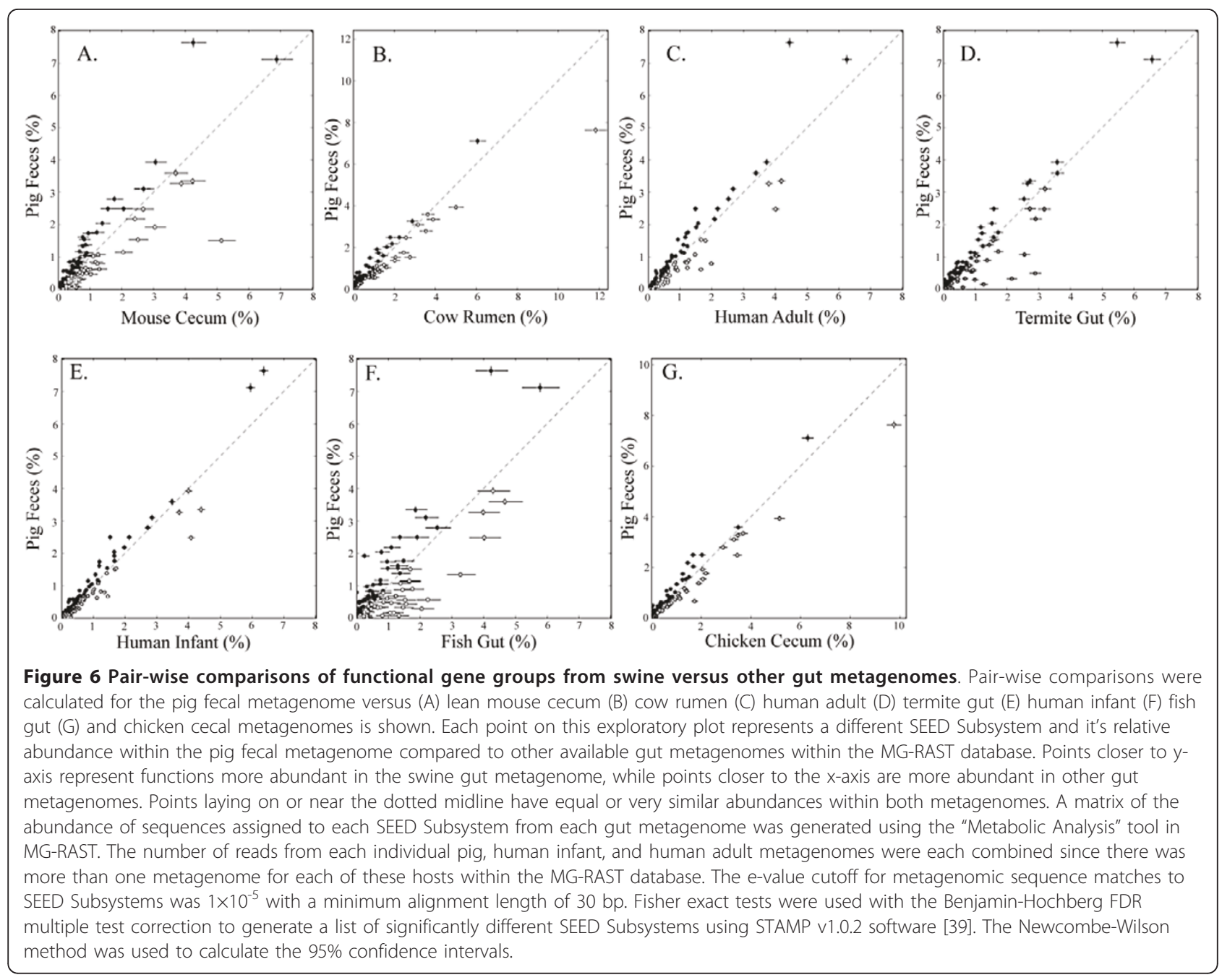

Pfams and COGs related to virulence factors such as adhesions were numerous within the gene families unique to the swine fecal metagenomes (Additional File 2, Table S6). Proteins involved in carbohydrate transport and attachment were both abundant and unique to the distal swine gut with more than 50 metagenomic contigs sharing high sequence homology to putative carbohydrate membrane transporters. Other proteins involved in carbohydrate metabolism were unique to the swine metagenome including glycosyl hydrolases, cellobiohydrolases, gluconolactonases, maltodextrin metabolism, and pectin lyases. The identification of unique gene families provides one line of evidence that the variable microbiome is a result of the microbial interaction with its surrounding environment. Because the environment surrounding gut microbes can vary among host species, a direct result of this level of functional diversity may be the generation of swine-specific microbiomes. Many proteins of unknown functions were also unique to the swine fecal metagenome, suggesting that some of them may be engaged in novel functions that have important biological meaning.

The high functional similarity between the pig and human metagenome is not surprising in light of the fact that they are mammalian omnivores with similar digestive tract structures and functions. Results from $16 \mathrm{~S}$ rRNA gene sequence analyses suggest that bacterial gut communities are similar among omnivorous mammals [2]. Similarities at the phylogenetic level between pig and human guts include the large presence of Firmicutes and members of the Bacteroidetes as the most abundant Gram-negative bacteria in their gastrointestinal tracts [14]. While differences in the relative abundance of Lactobacilli phylotypes have been noted, our data provides for the first time a functional perspective on how similar pigs and humans gut systems in spite of the differences in microbial community structure. In contrast, the functional similarities shared between the swine fecal metagenome and the termite gut was surprising and suggestive of previously unknown shared 
metabolic capabilities between these gut environments. For example, the pig and termite were the only two hosts possessing a suite of functions involved in archaeal lipid biosynthesis (Additional File 2, Fig. S13), suggesting an intimate relationship between the swine and archaeal gut populations [26]. Swine-specific methanogenic populations have been demonstrated in previous studies $[17,27]$. Similarities in cell wall and capsule profiles between the swine samples and termite gut may indicate that these functions can endow the swine gut with diversification of surface polysaccharide structures, allowing the host immune system to accommodate a diverse microbiota [2]. Presence of novel carbohydrate binding proteins and transporters also suggest the swine gut is capable of exploiting a diverse array of substrates.

Similarities in functional gene profiles (SEED subsystem abundance) among swine, chicken cecal and cow rumen metagenomes as compared to human gut metagenomes were unexpected considering the similarity shared between pig and human gut anatomy and physiology. These results suggest that that some microbial functions within the swine gut are shared among other agricultural animals, with arguably very different gastrointestinal anatomy and physiologies. For example, the elevated abundance of genes associated with protein turnover in pigs, chicken, and cow gut metagenomes is consistent with an increased use of amino acids for protein accretion in food production animals and is also consistent with the high protein diet fed to the pigs in this study. Additionally, the high abundance and diversity of carbohydrate utilization subsystems found in this swine metagenome may be a result of the high level of complex polysaccharides found in the diet. Altogether these data suggest that agricultural animal husbandry practices can impose significant selective pressures on the gut microbiota, regardless of gut type.

Surprisingly, this pig fecal metagenome revealed the presence of motile Treponema and Anaerovibrio genera. The presence of sequences associated with Treponema in this study (i.e., 3-4\% of all sequences swine fecal metagenome) suggests an order of magnitude higher abundance than a previous study in which swine gut microbiota revealed a very low abundance of Spirochetes using a culture independent method (i.e., $0.3 \%$ of all phylotypes) [14]. This genus has been previously detected in swine colonic samples but their presence in elevated levels is normally associated with swine dysentery. Discrepancies in community composition between cloning-based methods and noncloning based methods have been reported in the literature, primarily attributed to PCR amplification biases $[28,29]$. While many mammalian gut microbial communities are dominated by non-motile microbes, the termite hindgut and the fish gut harbor motile populations of bacteria, which are known to possess complex social behaviors [12,30,31]. This study revealed the pig gut may harbor previously unknown social dynamics, which may be relevant for maintaining compartmentalization and promoting niche selection within monogastric systems.

\section{Conclusions}

Herein, we report the first shotgun metagenomic pyrosequencing approach to study the microbiome of the swine distal gut. The overall goal of this study was to characterize the swine fecal microbiome with respect to species composition and functional content. Comparative metagenomic analyses identified unique and/or overabundant taxonomic and functional elements within swine distal gut microbiomes. These genetic attributes may help us better understand the microbial genetic factors that are relevant to swine health. Genes associated with the variable portion of gut microbiomes clustered by host environment with surprising hierarchical trends, suggesting that the variable microbiome content of a given host species may be reflective of the host ecology. While a larger metagenomic database that includes information on intra-host variation is needed for swine and other gut systems, this study provides a baseline for understanding the complexity of the swine gut microbial ecology, while also highlighting striking similarities and differences when compared to other animal gastrointestinal environments.

\section{Methods}

\section{Fecal Sample Collection}

Fecal samples were collected from eight, six-month old Yorkshire pigs from a large swine operation located in Northeastern Ohio, which housed more than 1,000 head of swine at the time of collection. Swine were weaned eight weeks after birth. Their diets consisted of a highenergy corn-soybean meal diet containing $14.00 \%$ crude protein, $0.63 \%$ lysine, $3.00 \%$ crude fat, $4.00 \%$ crude fiber, $0.55 \%-0.70 \%$ calcium, $0.52 \%$ phosphorus, $0.35 \%-0.50 \%$ salt, 0.3 ppm selenium, 80 ppm zinc. (Kalmbach Feeds, $\mathrm{OH})$. In addition, swine were supplemented with feed grade antibiotics for improvement in growth performance. Antibiotics consisted of chlortetracycline and penicillin at the concentration of $20 \mathrm{~g}$ per ton of feed. Fecal samples were transported to the laboratory on ice within four hours of collection, and stored at $-20^{\circ} \mathrm{C}$ until further processing. Fecal DNA was extracted with the FastDNA SPIN Kit (MP Biomedicals, Inc., Solon, OH) according to the manufacturer's instructions using 0.25 $\mathrm{g}$ of each fecal sample. Total DNA was quantified using a NanoDrop ${ }^{\circledR}$ ND-1000 UV spectrophotometer (NanoDrop Technologies, Wilmington, DE).

\section{Pyrosequencing and Gene Annotation}

A total of $24 \mu \mathrm{g}(3 \mu \mathrm{g}$ of each fecal DNA extract, $\mathrm{n}=8$ ) were pooled and sent for pyrosequencing to 454 Life 
Sciences, where two different sequencing runs were performed. The first run was performed using Genome Sequencer GS20 platform while the Genome Sequencer FLX instrument was used for the second run. Each pig fecal metagenomic sequencing run was assembled de novo using the Newbler assembly software by 454 Life Sciences. The metagenomes used in this paper are freely available from the SEED, JGI's IMG/M, and NCBI Short Read Archive. The NCBI genome project ID and GOLD ID for swine fecal GS20 and FLX metagenomic sequencing runs generated in this project are 39267 and Gm00197, respectively.

Raw sequencing reads from both datasets were submitted to the Joint Genome Institute's IMG/M-ER annotation pipeline using the proxygene method for gene annotation [4,32]. Additionally, both metagenome runs were annotated using the "Phylogenetic Analysis" tool within the MG-RAST pipeline [33]. The BLASTn algorithm (e-value less than $1 \times 10^{-5}$ and a sequence match length greater than 50 nucleotides) was used to identify small subunit rRNA genes from RDP [34], SILVA SSU [35], and Greengenes databases [36]. Within the MG-RAST pipeline, the "Metabolic Analysis" tool was used to search sequences from pig fecal metagenomes against the SEED database using the BLASTx algorithm (e-value less than $1 \times 10^{-5}$ and a sequence match length greater than 30 nucleotides) [37].

\section{Comparative Metagenomics and Statistical Analyses}

Comparative metagenomics was performed using both the IMG/M and MG-RAST pipelines. GS20 and FLX pig metagenomic runs were compared to the current publicly available gut metagenomes within each of these databases. Within the IMG/M pipeline, the two pig metagenomic runs were compared against three lean mouse (Mus musculus strain C57BL/6J) cecal metagenomes (Metagenome names: Mouse Gut Community lean1-3), two healthy human fecal metagenomes (Metagenome names: Human Gut Community Subject 7-8), and one termite (Nasutitermes $s p$ ) hindgut metagenome (Metagenome name: Termite hindgut). Descriptive information about these mouse, human and termite metagenomes can be found in the GOLD database under Gm00071, Gm00052, Gm00013 GOLD IDs, respectively. Within IMG/M the "Compare Genomes" tool was chosen to extract COG and Pfam protein profiles from the swine, mouse, human, and termite gut microbiomes. These profiles were then normalized for sequencing coverage by calculating the percent distribution, prior to downstream statistical analysis. To find over-abundant or unique functions to a given metagenomic dataset, a two-way hierarchical clustering of normalized COG and Pfam abundances was performed using the Bioinformatics Toolbox with Matlab version 2009a. Additionally, to determine if unique or overabundant functions were statistically meaningful, the binomial test within the Shotgun FunctionalizeR program was employed [38].

The GS20 and FLX pig fecal datasets were also compared against gut metagenomes available within the MG-RAST metagenomic annotation pipeline. The two pig fecal metagnonomic datasets were compared against the following MG-RAST metagenomic projects: cow rumen (Cow Rumen Project: 444168.3), chicken cecum (FS-CAP Project:4440285.3), human infant subjects In$A$, In-B, In-D, In-E, In- $M$ and In-R (Human Faeces Projects: 4440946.3, 4440945.3, 4440948.3, 4440950.3, 4440949.3, 4440951.3), human adult subjects F1-S, F1-T, F1-U, F2-V, F2-W, F2-X, and F2-Y (Human Faeces Projects: 4440939.9, 4440941.3, 4440940.3, 4440942.3, 4440943.3, 4440944.3, and 4440947.3), healthy fish gut (Fish Gut Project: 4441695.3), and lean mouse cecum (Human Faeces Project: 4440463.3). Within MG-RAST, phylogenetic information was extracted from these gut metagenomes using RDP [31], SILVA SSU [32], and Greengenes[33] databases (e-value less than $1 \times 10^{-5}$ and a sequence match length greater than 50 nucleotides). These taxonomic profiles were then normalized for differences in sequencing coverage by calculating percent distribution, prior to downstream statistical analysis. A non-parametric Wilcoxon exact test was used to statistically compare the taxonomic composition in any two metagenomes.

Additionally, within MG-RAST, the functional annotations (hits to SEED Subsystems) were extracted (e-value less than $1 \times 10^{-5}$ and a sequence match length greater than 50 nucleotides) to compare functional attributes across these gut metagenomes. In order to identify statistically significant and biologically meaningful differences between the swine gut and other endiobiotic microbiomes, we employed the two-way Fisher's exact test with a Benjamin-Hochberg FDR multiple test correction within STAMP v1.0.2 [39].

\section{Diversity Indices}

Observed richness, Chao1 estimator, abundance-based coverage estimator (ACE), jackknife estimator, and bootstrap estimator were used to evaluate community richness. Community diversity was described using Shannon, non-parametric Shannon, and Simpson indices within Mothur v 1.5.0 [40]. Sampling coverage was calculated using Good's coverage for the given operational taxonomic unit (OTU) definition, while the Boneh estimate was used to calculate the number of additional OTUs that would be observed for an additional 500 SSU reads. The aforementioned rRNA diversity indices and rarefaction curves were calculated using Mothur v 1.5.0 program with default parameters 
[40] and calculations for each index can found in the Mothur manual (http://www.mothur.org/wiki/ Mothur_manual). Functional diversity was assessed using SEED Subsystems [41], COG, and Pfam abundances from all available gut metagenomes. Diversity estimators used included Shannon-Weiner, Simpson's lambda, and Pielou's evenness analyses for measuring species richness and evenness. Functional diversity estimates, K- dominance plots, Principal Components Analysis, and clustering were performed using the PRIMER-E ecological software package [42].

\section{Additional material}

Additional file 1: Figures S1-S13. Fig. S1. Taxonomic distribution of viral sequences from swine feces. The percent of viral sequences retrieved from swine fecal GS20 (A) and FLX (B) metagenomes. Using the "Phylogenetic Analysis" tool within MG-RAST, the GS20 and FLX sequencing runs were searched against the SEED database using the BLASTx algorithm. The e-value cutoff for a hit to the database was $1 \times 10^{-}$ ${ }^{5}$ with a minimum alignment length of $30 \mathrm{bp}$. Fig. S2. Taxonomic distribution of bacterial orders from swine and other currently available gut microbiomes within MG-RAST. The percent of sequences assigned to each bacterial order from swine and other gut metagenomes is shown. Using the "Phylogenetic Analysis" tool within MG-RAST, each gut metagenome was searched against the RDP and greengenes databases using the BLASTn algorithm. The percentage of each bacterial order from swine, human infant, and human adult metagenomes were each averaged since there was more than one metagenome for each of these hosts within the MG-RAST database. The e-value cutoff for $16 \mathrm{~S}$ rRNA gene hits to the RDP and greengenes databases was $1 \times 10^{-5}$ with a minimum alignment length of $50 \mathrm{bp}$. Fig. S3. Taxonomic composition of bacterial genera using $16 \mathrm{~S}$ rDNA sequences retrieved from swine fecal metagenomes. The percent of sequences assigned to each of the bacterial genera from the pig fecal GS20 (A) and FLX (B) metagenomes is shown. Using the "Phylogenetic Analysis" tool within MG-RAST, the GS20 and FLX pig fecal metagenomes were searched against the RDP and greengenes databases using the BLASTn algorithm. The e-value cutoff for $16 \mathrm{~S}$ rRNA gene hits to the databases was $1 \times 10^{-5}$ with a minimum alignment length of $50 \mathrm{bp}$. Fig. S4. Dominance profiles of swine and other gut metagenomes available within MG-RAST. K-dominance plots were calculated based on the abundance of gut metagenomic sequences assigned at the RDP genus level taxonomy using the "Phylogenetic Analysis" tool within MG-RAST. The e-value cutoff for 165 rRNA gene hits to the RDP database was $1 \times 10^{-5}$ with a minimum alignment length of $50 \mathrm{bp}$. K-dominance for each of the individual gut metagenomes was calculated using PRIMER-E v6 software [42]. Fig. S5. Rarefaction curves for $16 \mathrm{~S}$ rRNA gene sequences from swine and other gut metagenomes. Rarefaction curves were calculated based on the observed abundance of gut metagenomic sequences assigned at the RDP genus level taxonomy using MG-RAST's "Phylogenetic Analysis" tool. The e-value cutoff for $16 \mathrm{~S}$ rRNA gene hits to the RDP database was $1 \times 10^{-5}$ with a minimum alignment length of $50 \mathrm{bp}$. Rarefaction curves for each gut metagenome were calculated within Mothur $\vee$ 1.5.0 software using default parameters [40]. Rarefaction curves provide a way of comparing the richness observed in these different gut metagenomic samples. Fig. S6. Functional composition of the swine fecal microbiome. The percent of GS20 (A) and FLX (B) swine fecal metagenomic sequences assigned to general SEED Subsystems is shown. Using the "Metabolic Analysis" tool within MG-RAST, the GS20 and FLX pig fecal sequencing runs were searched against the SEED database using the BLASTx algorithm. The e-value cutoff for metagenomics sequence matches to the SEED Subsystem database was $1 \times 10^{-5}$ with a minimum alignment length of $30 \mathrm{bp}$. Fig. S7. Comparison of functional composition of swine and other currently available gut metagenomes within the MG-RAST pipeline. Percentage of gut metagenomic sequences assigned to general SEED Subsystems is shown. Using the "Metabolic Analysis" tool within MG-RAST, gut metagenomes were searched against the SEED database using the BLASTx algorithm. The percentage of each general SEED Subsystem from swine, human infant, and human adult metagenomes were each averaged since there was more than one metagenome for each of these hosts within the MG-RAST database. The e-value cutoff for metagenomic sequence matches to the SEED Subsystem database was $1 \times 10^{-5}$ with a minimum alignment length of 30 bp. Fig. S8. Percent distribution of prophage and DNA recombination genes from gut metagenomes available within the MG-RAST pipeline. Using the "Metabolic Analysis" tool within MG-RAST, the available gut metagenomes were searched against the SEED database using the BLASTx algorithm. Percentage contribution of each gut metagenome assigned to functional classes within "Prophage/DNA recombination" SEED Subsystem is shown. The e-value cutoff for metagenomic sequences matches to this SEED Subsystem was $1 \times 10^{-5}$ with a minimum alignment length of $30 \mathrm{bp}$. Fig. S9. Hierarchical clustering of gut metagenomes available within MG-RAST based on the relative abundance of cell wall and capsule genes. A matrix consisting of the number of reads assigned to genes within the "Cell wall and Capsule" SEED Subsystem from each gut metagenome was generated using the "Metabolic Analysis" tool within MG-RAST. The e-value cutoff for metagenomic sequences matches to this SEED Subsystem was $1 \times 10^{-5}$ with a minimum alignment length of $30 \mathrm{bp}$. Resemblance matrices were calculated using Bray-Curtis dissimilarities within PRIMER v6 software [41]. Clustering was performed using the complete linkage algorithm. Dotted branches denote that no statistical difference in similarity profiles could be identified for these respective nodes, using the SIMPROF test within PRMERv6 software. Fig. S10. Transposases derived from gut metagenomes available within JGI's IMG/M database. The percent of total annotated tranposase gene families from pig, mouse, human, and termite gut metagenomes is shown. The percentage of each transposase family from swine, human, and mouse gut metagenomes were each averaged since there was more than one metagenome for each of these hosts within the JGI's IMG/M database. Metagenomic sequences were assigned to transposase gene families using the IMG 2.8 pipeline. Fig. S11. Composition of resistance genes present with the swine fecal metagenome. The percent of swine fecal metagenomic sequences assigned to the "Resistance to Antibiotics and Toxic Compounds" SEED Subsystem is shown. The number of GS20 and FLX assigned to genes within this SEED Subsystem were combined. The e-value cutoff for metagenomic sequence matches to this SEED Subsystem database was $1 \times 10^{-5}$ with a minimum alignment length of $30 \mathrm{bp}$. Fig. S12. Differential functions within the swine fecal metagenome. A list of significantly different SEED Subsystems and their relative abundance are shown for pair-wise comparisons of the pig fecal metagenome versus other available gut metagenomes within the MG-RAST database. A matrix of the abundance of sequences assigned to each SEED Subsystem from each gut metagenome was generated using the "Metabolic Analysis" tool in MG-RAST. The number of reads from each individual pig, human infant, and human adult metagenomes were each combined since there was more than one metagenome for each of these hosts within the MGRAST database. The e-value cutoff for metagenomic sequence matches to SEED Subsystems was $1 \times 10^{-5}$ with a minimum alignment length of 30 bp. Pair-wise comparisons of pig fecal metagenomes versus (A) Lean Mouse cecum (B) Cow rumen (C) Fish gut (D) Termite gut (E) Chicken cecum (F) Human adult (G) Human infant gut metagenomes are shown. Fisher exact tests were employed using the Benjamin-Hochberg FDR multiple test correction to generate a list of significantly different SEED Subsystems using STAMP V1.0.2 software [39]. Significantly different SEED Subsystems with a q-value less than $1 \times 10^{-5}$ are shown. Significantly different SEED Subsystems from the pig fecal metagenome are shown in blue and all other gut metagenomes are shown in orange. Fig. S13. Comparison of lipid biosynthesis genes from gut metagenomes available within the MG-RAST pipeline. Using the "Metabolic Analysis" tool within MG-RAST, the gut metagenomes were searched against the SEED database using the BLASTx algorithm. Percentage of gut metagenomic reads assigned to genes in the "Fatty Acid and Lipid Biosynthesis" SEED Subsystem is shown. The e-value cutoff for metagenomics sequence matches to this SEED Subsystem database was $1 \times 10^{-5}$ with a minimum alignment length of $30 \mathrm{bp}$. 
Additional file 2: Tables S1-S6. Table S1. The results of a Wilcoxon test to compare taxonomic distribution of bacterial orders from endobiotic microbiomes. Table S2. Binomial test for comparing abundance of bacteria phyla from distal gut metagenomes. Table S3. Binomial test for comparing abundance of bacteria genera from distal gut metagenomes. Table S4. Diversity analyses for endobiotic metagenomes using SEED Subsystem annotations. Table S5. Diversity analyses for endobiotic metagenomes using COG and Pfam annotations. Table S6. Pfams and COGs unique to swine fecal metagenomes.

\section{Acknowledgements}

The U.S. Environmental Protection Agency, through its Office of Research and Development, funded and managed, or partially funded and collaborated in, the research described herein. It has been subjected to the Agency's administrative review and has been approved for external publication. Any opinions expressed in this paper are those of the author(s) and do not necessarily reflect the views of the Agency, therefore, no official endorsement should be inferred. Any mention of trade names or commercial products does not constitute endorsement or recommendation for use. This work was also partly funded by the United States Environmental Protection Agency Traineeship and National Science Foundation grant to DBO.

\section{Author details}

'University of Cincinnati, Department of Civil and Environmental Engineering, Cincinnati, OH 45220, USA. ${ }^{2}$ National Risk Management Research Laboratory, U. S. Environmental Protection Agency, Cincinnati, OH 45268, USA. ${ }^{3}$ National Exposure Research Laboratory, U. S. Environmental Protection Agency, Cincinnati, OH 45268, USA. ${ }^{4}$ Lawrence Berkeley National Laboratory, Earth Sciences Division, Berkeley, CA 94720, USA. ${ }^{5}$ Department of Civil and Environmental Engineering, Missouri University of Science and Technology, Rolla, MO 65409, USA.

\section{Authors' contributions}

RL carried out sample collection, sample processing, bioinformatic analyses, and manuscript preparation. JSD conceived of the study, and participated in its design and coordination and helped to draft the manuscript. SG participated in bioinformatic and statistical analyses. JM participated in bioinformatic analyses. DBO participated in design and coordination of the study and helped revise the manuscript. All authors read and approved the final manuscript.

Received: 13 September 2010 Accepted: 15 May 2011

Published: 15 May 2011

\section{References}

1. Ley RE, Peterson DA, Gordon J: Ecological and evolutionary forces shaping microbial diversity in the human intestine. Cell 2006, 124:837-848.

2. Ley RE, Hamady M, Lozupone C, Turnbaugh PJ, Ramey RR, Bircher JS, Schlegel ML, Tucker TA, Schrenzel MD, Knight R, Gordon Jl: Evolution of mammals and their gut microbes. Science 2008, 320:1647-1651.

3. Hugenholtz P, Tyson GW: Microbiology metagenomics. Nature 2008, 455:481-483

4. Markowitz VM, Ivanova N, Szeto E, Palaniappan K, Chu K, Dalevi D, Chen IM, Grechkin Y, Dubchak I, Anderson I, Lykidis A, Mavromatis K, Hugenholtz P, Kyrpides NC: IMG/M: a data management and analysis system for metagenomes. Nucleic Acids Res 2008, 36:D534-D538.

5. Kurokawa K, Itoh T, Kuwahara T, Oshima K, Toh H, Toyoda A, Takami H, Morita H, Sharma VK, Srivastava TP, Taylor TD, Noguchi H, Mori H, Ogura Y, Ehrlich DS, Itoh K, Takagi T, Sakaki Y, Hayashi T, Hattori M: Comparative metagenomics revealed commonly enriched gene sets in human gut microbiomes. DNA Res 2007, 14:169-181.

6. Brulc JM, Antonopoulos DA, Miller ME, Wilson MK, Yannarell AC, Dinsdale EA, Edwards RE, Frank ED, Emerson JB, Wacklin P, Coutinho PM, Henrissat B, Nelson KE, White BA: Gene-centric metagenomics of the fiber-adherent bovine rumen microbiome reveals forage specific glycoside hydrolases. Proc Natl Acad Sci 2009, 106:1948-1953.
7. Dinsdale EA, Edwards RA, Hall D, Angly F, Breitbart M, Brulc JM, Furlan M, Desnues C, Haynes M, Li L, McDaniel L, Moran MA, Nelson KE, Nilsson C, Olson R, Paul J, Brito BR, Ruan Y, Swan BK, Stevens R, Valentine DL, Thurber RV, Wegley L, White BA, Rohwer F: Functional metagenomic profiling of nine biomes. Nature 2008, 452:629-632.

8. Qu A, Brulc JM, Wilson MK, Law BF, Theoret JR, Joens LA, Konkel ME, Angly F, Dinsdale EA, Edwards RA, Nelson KE, White BA: Comparative metagenomics reveals host specific metavirulomes and horizontal gene transfer elements in the chicken cecum microbiome. PLOS ONE 2008, 3: e2945.

9. Tringe SG, von Mering C, Kobayashi A, Salamov AA, Chen K, Chang HW, Podar M, Short JM, Mathur EJ, Detter JC, Bork P, Hugenholtz P, Rubin EM: Comparative metagenomics of microbial communities. Science 2005, 308:554-557.

10. Turnbaugh PJ, Ley R, Mahowald M, Magrini V, Mardis E, Gordon J: An obesity-associated gut microbiome with increased capacity for energy harvest. Nature 2006, 444:1027-1031.

11. Turnbaugh PJ, Ley RE, Hamady M, Fraser-Liggett CM,R, Knight R, Gordon Jl: The Human Microbiome Project. Nature 2007, 449:804-810.

12. Warnecke $F$, Luginbuhl $P$, Ivanova $N$, Ghassemian $M$, Richardson $T H$, Stege JT, Cayouette M, McHardy AC, Djordjevic G, Aboushadi N, Sorek R, Tringe SG, Podar M, Martin HG, Kunin V, Dalevi D, Madejska J, Kirton E, Platt D, Szeto E, Salamov A, Barry K, Mikhailova N, Kyrpides NC, Matson EG, Ottesen EA, Zhang XN, Hernandez M, Murillo C, Acosta LG: Metagenomic and functional analysis of hindgut microbiota of a wood-feeding higher termite. Nature 2007, 450:560-565.

13. Castillo M, Skene G, Roca M, Anguita M, Badiola I, Duncan SH, Flint HJ, Martín-Orúe SM: Application of 16S rRNA gene-targetted fluorescence in situ hybridization and restriction fragment length polymorphism to study porcine microbiota along the gastrointestinal tract in response to different sources of dietary fibre. FEMS Microbiol Ecol 2007, 59:138-146.

14. Leser TD, Amenuvor JZ, Jensen TK, Lindecrona RH, Boye M, Moller K: Culture-independent analysis of gut bacteria: the pig gastrointestinal tract microbiota revisited. Appl Environ Microbiol 2002, 68:673-690.

15. Lin C, Markowitz LVM, Mavromatis K, Ivanova NN, Chen IM, Chu K, Kyrpides NC: IMG ER: a system for microbial genome annotation expert review and curation. Bioinformatics 2009, 25:2271-2278.

16. Snell-Castro R, Godon JJ, Delgenès JP, Dabert P: Characterisation of the microbial diversity in a pig manure storage pit using small subunit rDNA sequence analysis. FEMS Microbiol Ecol 2005, 52:229-242.

17. Lamendella R, Santo Domingo JW, Yannarell AC, Ghosh S, Di Giovanni G, Mackie RI, Oerther DB: Evaluation of swine-specific PCR assays used for fecal source tracking and analysis of molecular diversity of swinespecific "Bacteroidales" populations. Appl Environ Microbiol 2009, 75:5787-5796.

18. Jensen BB: Methanogenesis in monogastric animals. Environ Monit Assess 1996, 42:99-112.

19. Fangman TJ, Hardin LE, Grellner G, Carlson MS, Zulovich JM, Coleman JL: Performance and disease status of pigs grown in a wean-to-finish facility compared to pigs grown in a conventional nursery and growerfinisher facility. I Swine Health Prod 2001, 9:71-76.

20. USDA National Animal Health Monitoring System: Part I. Reference of Swine Health and Management in the United States United States; 2001.

21. Taylor NM, Clifton-Hadley FA, Wales AD, Ridley A, Davies RH: Farm-level risk factors for fluoroquinolone resistance in E. coli and thermophilic Campylobacter spp. on finisher pig farms. Epidemiol Infect 2009, 137:1121-1134.

22. van den Broek IV, van Cleef BA, Haenen A, Broens EM, van der Wolf PJ, van den Broek MJ, Huijsdens XW, Kluytmans JA, Van de Giessen AW, Tiemersma EW: Methicillin-resistant Staphylococcus aureus in people living and working in pig farms. Epidemiol Infect 2009, 137:700-708.

23. Li XZ, Nikaido H, Poole K: Role of mexA-mexB-oprM in antibiotic efflux in Pseudomonas aeruginosa. Antimicrob Agents Chemother 1995, 39:1948-1953.

24. Boneca IG: The role of peptidoglycan in pathogenesis. Curr Opinion Microbiol 2005, 8:46-53.

25. Lindemann MD: Supplemental folic acid: a requirement for optimizing swine reproduction. Journal of Animal Science 1993, 71:239-246.

26. Ufnar JA, Ufnar DF, Wang SY, Ellender RD: Development of a swinespecific fecal pollution marker based on host differences in methanogen mcrA genes. Appl Environ Microbiol 2007, 73:5209-17. 
27. Boucher Y, Kamekura M, Doolittle WF: Origins and evolution of isoprenoid lipid biosynthesis in archaea. Mol Microbiol 2004, 52:515-527.

28. Webster G, Newberry CJ, Fry JC, Weightman AJ: Assessment of bacterial community structure in the deep sub-seafloor biosphere by $16 \mathrm{~S}$ rDNAbased techniques: a cautionary tale. J Micro Methods 2003, 55:155-164.

29. Wilson KH, Wilson WJ, Radosevich JL, DeSantis TZ, Viswanathan VS, Kuczmarski TA, Andersen GL: High-density microarray of small-subunit ribosomal DNA probes. Appl Environ Microbiol 2002, 68:2535-2541.

30. Ingham CJ, Ben-Jacob E: Swarming and complex pattern formation in Paenibacillus vortex studied by imagine and tracking cells. $B M C$ Microbiology 2008, 8:36.

31. Kirchhof G, Eckert BBM, Stoffels MJl, Baldani JI VM, Reis VM, Hartmann A: Herbaspirillum frisingense sp. nov., a new nitrogen-fixing bacterial species that occurs in C4-fibre plants. Int I Syst Evol Microbiol 2001, 51:157-168.

32. Dalevi D, Ivanova NN, Mavromatis K, Hooper S, Szeto E, Hugenholtz P, Kyrpides NC, Markowitz VM: Annotation of metagenome short reads using proxygenes. Bioinformatics 2008, 24:17-13.

33. Meyer F, Paarmann D, D'Souza M, Olson R, Glass EM, Kubal M, Paczian T, Rodriguez A, Stevens R, Wilke A, Wilkening J, Edwards RA: The Metagenomics RAST server - A public resource for the automatic phylogenetic and functional analysis of metagenomes. BMC Bioinformatics 2008, 9:386.

34. Cole JR, Chai B, Farris RJ, Wang Q, Kulam-Syed-Mohidee AS, McGarrell DM, Bandela AM, Cardenas E, Garrity GM, Tiedje JM: The ribosomal database project (RDP-II): introducing myRDP space and quality controlled public data. Nucleic Acids Res 2007, 35:169-172

35. Pruess E, Quast C, Knittel K, Fuchs B, Ludwig W, Peplies J, Glöckner FO: SILVA: a comprehensive online resource for quality checked and aligned ribosomal RNA sequence data compatible with ARB. NuC Acids Res 2007, 35:7188-7196

36. DeSantis TZ, Hugenholtz $\mathrm{P}$, Larsen N, Rojas M, Brodie EL, Keller K, Huber T, Dalevi D, Hu P, Andersen GL: Greengenes, a chimera-checked 16S rRNA gene database and workbench compatible with ARB. Appl Environ Microbiol 2006, 72:5069-5072.

37. Altschul SF, Gish W, Miller W, Myers EW, Lipman DJ: Basic local alignment search tool. J Mol Biol 1990, 215:403-410

38. Kristiansson E, Hugenholtz P, Dalevi D: ShotgunFunctionalizeR: An Rpackage for functional analysis of metagenomic data. Bioinformatics 2009, 25:2737-2738.

39. Parks DH, Beiko RG: Identifying biologically relevant differences between metagenomic communities. Bioinformatics 2010, 26:715-721.

40. Schloss PD, Westcott SL, Ryabin T, Hall JR, Hartmann M, Hollister EB, Lesniewski RA, Oakley BB, Parks DH, Robinson CJ, Sahl JW, Stres B, Thallinger GG, Van Horn DJ, Weber CF: Introducing mothur: open source, platform-independent, community-supported software for describing and comparing microbial communities. Appl Environ Microbiol 2009, 75:7537-41.

41. Overbeek R, Begley T, Butler RM, Choudhuri JV, Chuang HY, Cohoon M, de Crécy-Lagard V, Diaz N, Disz T, Edward R, Fonstein M, Frank ED, Gerdes S, Glass EM, Goesmann A, Hanson A, Iwata-Reuyl D, Jensen R, Jamshidi N, Krause L, Kubal M, Larsen N, Linke B, McHardy AC, Meyer F, Neuweger H, Olsen G, Olson R, Osterman A, Portnoy V, Pusch GD, Rodionov DA, Rückert C, Steiner J, Stevens R, Thiele I, Vassieva O, Ye Y, Zagnitko O, Vonstein $\mathrm{V}$ : The subsystems approach to genome annotation and its use in the project to annotate 1000 genomes. Nucleic Acids Res 2005, 33:5691-702.

42. Clarke KR, Gorley RN: PRIMER-E. PRIMER-E Ltd, Plymout, UK; 2001

doi:10.1186/1471-2180-11-103

Cite this article as: Lamendella et al:: Comparative fecal metagenomics unveils unique functional capacity of the swine gut. BMC Microbiology 2011 11:103.

\section{Submit your next manuscript to BioMed Central and take full advantage of:}

- Convenient online submission

- Thorough peer review

- No space constraints or color figure charges

- Immediate publication on acceptance

- Inclusion in PubMed, CAS, Scopus and Google Scholar

- Research which is freely available for redistribution

Submit your manuscript at www.biomedcentral.com/submit
C Biomed Central 